\title{
Apoptotic Find-me Signals are an Essential Driver of Stem Cell Conversion To
} The Cardiac Lineage

Vanderbilt University School of Medicine

Nashville TN 37240

$11{ }^{*}$ Contact information:

12 lan.g.macara@vanderbilt.edu

13 615-875-5565

\section{Abstract}

16 Pluripotent stem cells can be driven by manipulation of Wnt signaling through a series of states

17 similar to those that occur during early embryonic development, transitioning from an epithelial phenotype into the cardiogenic mesoderm lineage and ultimately into functional cardiomyocytes ${ }^{1-4}$. Strikingly, we observed that induced pluripotent stem cells (iPSCs) and embryonic stem cells (ESCs) undergo widespread apoptosis upon Wnt activation, followed by a synchronous epithelial-mesenchymal transition (EMT). The EMT requires induction of transcription factors

22 SNAI1/SNAI2 downstream of MESP1 expression, and double knock-out of SNA/1/2, or loss of

23 MESP1 in iPSCs blocks EMT and prevents cardiac differentiation. Remarkably, blockade of

24 early apoptosis chemically or by ablation of pro-apoptotic genes also completely prevents the

25 EMT, suppressing even the earliest events in mesoderm conversion, including EOMES, TBX6, 26 and MESP1 induction. Conditioned medium from WNT-activated WT iPSCs overcomes the

27 block to EMT by cells incapable of apoptosis (Apop-), suggesting the involvement of soluble factors from apoptotic cells in mesoderm conversion. Treatment with a purinergic P2Y receptor inhibitor or addition of apyrase demonstrated a requirement for nucleotide triphosphate signaling. ATP was sufficient to induce a partial EMT in Apop- cells treated with WNT activator.

31 We conclude that nucleotides, in addition to acting as chemo-attractants for clearance of

32 apoptotic cells can, unexpectedly, function as essential paracrine signals in mesoderm

33 specification. 


\section{Main}

A mesenchymal-epithelial transition (MET) is an early and essential event during reprogramming by the Yamanaka factors SOX2, OCT4, C-MYC and KLF4 ${ }^{5-7}$. During reprogramming of mouse fibroblasts, SOX2 and OCT4 suppress SNAI1/2 and ZEB1/2, which are key transcription factors that promote the reverse process, an epithelial-mesenchymal transition (EMT), while c-MYC represses TGF $\beta$ signaling and KLF4 induces epithelial gene expression ${ }^{8,9}$. EMT occurs during gastrulation as epithelial epiblast cells ingress through the primitive streak ${ }^{10}$. EMT is obligatory for ESCs to differentiate into definitive endoderm ${ }^{11}$, while a sequential EMT/MET occurs during the conversion of the hepatocyte lineage ${ }^{12}$. However, the reason why these processes are required for reprogramming and differentiation remains unknown.

\section{Initiation of cardiomyocyte specification triggers apoptosis followed by an EMT} Human iPSCs (GM25256) used in this study expressed appropriate pluripotency markers (NANOG, OCT4, SOX2) and epithelial polarity proteins including SCRB, LLGL2, PAR-3, PKCzeta and PALS1 (Supplementary Fig S1A). They assemble ZO-1 positive tight junctions (TJ) and E-cadherin positive adherens junctions (AJ), localize polarity proteins appropriately, and form cell monolayers with a cobblestone appearance (Fig 1A). Treatment with a WNT activator (CHIR 99021) for 48 hrs followed by treatment with a WNT signaling inhibitor (Fig 1B) was used to drive cardiomyocyte specification ${ }^{2}$. This protocol promoted exit from pluripotency and the sequential induction of mid-primitive streak, marked by induction of EOMES and TBXT (Bra/T) from 9 - $36 \mathrm{hrs;} \mathrm{lateral} \mathrm{mesoderm,} \mathrm{marked} \mathrm{by} \mathrm{induction} \mathrm{of} \mathrm{TBX6} \mathrm{and} \mathrm{MESP1} \mathrm{from} 36$ - $48 \mathrm{hrs,}$ and cardiac mesoderm, marked by induction of ISL1, NKX2.5 and ANP, for $48-72 \mathrm{hrs}$ (Supplementary Fig S1B, C). Consistent with previous data ${ }^{1,2}$, spontaneous beating of immature cardiomyocytes was observed after 10-12 days (Supplementary Video 1). Cardiac specification was accompanied by loss of E-cadherin, and expression of Slug and Vimentin (Supplementary Fig S1D-E).

Strikingly, early induction of cardiomyocyte specification resulted in extensive cell extrusion (Fig 1C and Supplementary Video 2), caused by apoptosis that began within 12 hours, and slowly diminished over 2 days, as measured by cleaved caspase 3, cleaved PARP, and Annexin exposure (Fig $1 \mathrm{D}-\mathrm{H}$ ). This was followed at $49-50 \mathrm{hrs}$ by an abrupt wave of intercellular junction disassembly that occurred throughout the culture and was complete within $\sim 2$ hrs (Fig 1l; Supplementary video 2). Cell-cell contacts and cortical actin were lost, with acquisition of stress fibers and a spindle-shaped morphology (Fig $1 \mathrm{I}, \mathrm{J})$. Surprisingly, expression of the AJ-marker E- 
cadherin did not diminish until $\sim 24 \mathrm{hrs}$ after junction disassembly (Fig 1K). Nonetheless, other markers of EMT were detected, including Snail and Slug, and the mesenchymal marker vimentin (Fig 1K, Supplementary Fig S1 D-E). Importantly, both apoptosis and EMT also occurred in hESCs that were driven along the cardiac mesoderm lineage, with increased PARP cleavage over the initial 2 days (Supplementary Fig S1F-G), followed by increased Snail and Slug (Supplementary Fig S1H). Importantly, the extensive apoptosis we observed is specific to Wnt activation, as treatment with retinoic acid does not stimulate cell death ${ }^{13}$.

77 The global EMT occurs almost immediately after addition of IWP-2 inhibitor in the standard 78 protocol for cardiomyocyte induction (Fig 1I-K). However, the causal relationship between these 79 two events was unclear. Therefore, we initiated mesoderm induction by addition of CHIR but 80 withheld inhibitor at $48 \mathrm{hrs}$ and did not remove the CHIR. Notably, the EMT still occurred on 81 schedule in these cells (Fig $1 \mathrm{~L}$ and Supplementary Fig S1I), demonstrating that it is independent of WNT inhibition and that, with astonishing fidelity, the iPSCs can synchronously time the EMT to a $1-2 \mathrm{hr}$ window 2 days after WNT activation.

\section{EMT is driven by the induction of SNAI1 and SNAI2, downstream of MESP1}

86 To investigate the mechanism of EMT, we first used RT-qPCR to analyze SNA/1/2 induction and found that SNAl1 peaks at $\sim 50 \mathrm{hrs}$ while SNAI2 peaks at about $72 \mathrm{hrs}$ (Fig 2A), as also detected by immunoblot (Fig. 1K): We next asked if the observed EMT is dependent on SNAI1 and/or SNAI2, by CRISPR/Cas9-mediated gene editing in the iPSCs (Fig 2 B,C). Knockout of either gene alone had little effect because of compensatory induction of the remaining gene (data not shown), but a double knockout (DKO) of SNAl1/2 efficiently blocked the EMT (Fig 2D).

92 Moreover, these cells did not continue down the cardiac mesoderm lineage. They did not

93 express key cardiac markers such as NKX2.5 and cTNT and showed a reduced expression of GATA4 (Fig. 2E). We conclude that the EMT induced in response to WNT activation in iPSCs is driven by expression of Snail and Slug, and that these factors are essential for specification of the cardiac lineage.

$97 M E S P 1$ is a pioneer cardiac factor in vivo. During embryonic stem cell differentiation, MESP1 is expressed in ESC-derived cardiac mesoderm progenitors, and is required for cardiac mesoderm specification $^{14}$. Moreover, MESP1 regulates expression of multiple EMT-promoting genes 100 including SNA/1/2. Consistent with these data, we found that CRISPR/Cas9-mediated KO of 101 MESP1 (Fig 2F) prevented induction of SNAI1/2 (Fig 2F), blocked the scheduled EMT at 49hrs 102 post-addition of CHIR (Fig 2G), suppressed expression of NKX2.5, HAND1, cTNT and GATA4 103 (Fig 2H) and prevented differentiation into cardiomyocytes (Supp.Video 3). We conclude that 
MESP1 during mesoderm conversion induces SNA/1/2, which in turn drive a synchronous EMT that is essential for further differentiation along the cardiac lineage.

\section{Apoptosis is an essential antecedent to SNAI1/SNAI2 induction and EMT}

The initial CHIR treatment of iPSCs caused a rapid, widespread apoptosis, as detected by cleaved caspase 3, PARP cleavage, and Annexin exposure (Fig 1C-H). During this time surviving cells continued to proliferate, increasing the overall cell density (Fig 3A, Supp. Fig S3G-H).

To test whether apoptosis has any impact on EMT and conversion along the cardiac mesoderm lineage ,we blocked cell death in cultures treated with CHIR, using the pan-caspase inhibitor QVD-OPH. Remarkably, this drug totally prevented the scheduled EMT (Fig. 3A). The cells maintained a cobblestone appearance, retained tight junctions, grew to high density (Supp. Fig S3G), and failed to express Snail or Slug (Fig 3A-C). Inhibition of apoptosis was marked by loss of cleaved Caspase 3 and PARP (Fig 3B). A similar effect of Q-VD-OPH on EMT was observed in human ESCs (Supp. Fig S3A-C).

To validate the connection to cell death we generated iPSC lines deleted for the apoptotic executioner proteases Caspase 3 or Caspase 9, using CRISPR/Cas9-mediated gene editing (Supp. Fig S3D). These lines did not proliferate as rapidly as the parental WT or the control nontarget (NT) iPSCs, but nonetheless formed island cultures. Notably, treatment with CHIR did not cause TJ disassembly, even after prolonged incubation, and did not induce SNAI1/2 (Supp. Fig 3E-F).

Next, to prevent the apoptotic events upstream of caspase activation, we deleted the proapoptotic genes $B A X$ and $B A K^{15}$. Unlike the Caspase 3 and $9 \mathrm{KO}$ lines, these double knockout (DKO) cells grew at rates comparable to control cells (Supp. Fig S3H), but again, no EMT was detected after treatment with CHIR as determined by persistence of TJs (Fig 3D) and by the absence of Snail and Slug expression at both the protein and mRNA levels (Fig 3E - G). Moreover, cardiomyocyte differentiation was also blocked (Fig 3H; Supp. Fig $3 \mathrm{I}$ and Supp.Video 4). Similarly, addition of Q-VD-OPH during both, CHIR-99021 and IWP-2 steps, blocks induction of the cardiac marker HAND1 (Supp. Fig S3J).

Together, these data strongly support an unanticipated requirement for apoptosis, induced after WNT activation, to permit the entry of pluripotent cells into the mesoderm lineage. We note that cell death has been observed in early mouse embryos, just prior to gastrulation and in primitive streak, although its function is unknown ${ }^{16}$. 


\section{Apoptosis is required very early in selection of the mesoderm lineage}

To determine at which stage apoptosis permits pluripotent stem cells to differentiate towards

140 cardiomyocytes, we treated BAX/BAK DKO iPSCs with CHIR, then harvested cells at $46 \mathrm{hrs}$ for

141 QRT-PCR. Surprisingly, even very early changes in gene expression, including TBXT (T/Bra)

142 and EOMES were drastically reduced or suppressed. A similar suppression was caused by the

143 treatment of WT iPSCs with QVD-OPH prior to addition of CHIR (Fig 4B). The failure to express

144 these genes was confirmed by immunofluorescence (Fig. 4C) and by immunoblotting for T/Bra

145 (Fig. 4D). Later changes associated with mesoderm induction, including TBX6 and MESP1

146 were also inhibited (Fig. 4A-B); however, expression of the pluripotent marker Nanog decreased

147 on schedule (Fig 4E). Importantly, DKO iPSCs lacking BAX and BAK can still enter the neuronal

148 lineage ${ }^{15}$.

149 These data suggest that, even though apoptosis continues to occur for $>40$ hours after

150 activation of WNT signaling, it is necessary for a very early event following initiation of

151 mesoderm specification. To further test this hypothesis, we treated WT iPSCs with CHIR and

152 added Q-VD-OPH only for the first $24 \mathrm{hrs}$, after which time the medium was replaced to $\mathrm{CHIR}$

153 alone for an additional $48 \mathrm{hrs}$ (Fig 4F) or, as a control, iPSCs were treated with CHIR alone until

$15424 \mathrm{hrs}$ had passed, then incubated with CHIR + Q-VD-OPH (Supp. Fig S4C). Notably, early

addition of this inhibitor completely blocked the subsequent EMT and strongly reduced

Snail/Slug induction (Fig. 4G-H); but addition after $24 \mathrm{hrs}$ had no effect and EMT occurred on schedule (Supp. Fig S4C-D)). Together, these data highlight an unanticipated essential role for apoptosis in the initial steps towards mesoderm specification.

\section{A soluble factor from apoptotic cells permits pluripotent cells to enter the mesoderm}

\section{1 lineage}

162 How might apoptosis allow pluripotent stem cells to enter the cardiac mesoderm lineage? We considered two possibilities. First, cell death might relax the space constraints between cells in a colony, allowing them to stretch and generate tension, which in principle could activate signaling through YAP/TAZ or some other pathway to promote mesoderm conversion. Alternatively, apoptotic cells might release a soluble factor that promotes mesoderm conversion.

167 We discounted the first hypothesis as unlikely, because we did not detect any increase in 168 nuclear YAP localization after CHIR treatment (Supp. Fig S5A). To test the second hypothesis, 169 we treated both WT and the BAK/BAX DKO iPSCs for $24 \mathrm{hrs}$ with CHIR then replaced the DKO 170 medium with conditioned medium (CM) from the apoptosing WT iPSCs, and continued to 171 incubate the DKO cells for a further $48 \mathrm{hrs}$ (Fig 5A). Remarkably, the DKO cells receiving the 
172 CM underwent a dramatic EMT (Fig 5B). To test if the CM also relieved the blockade to

173 expression of mesoderm lineage genes caused by the inability of the DKO cells to undergo

174 apoptosis, we performed RT-qPCR and found significant increases in EOMES, MESP1, TBX6,

175 and SNAI1/2 (Fig 5C). These results clearly demonstrate that a soluble factor released by

176 WNT-induced apoptosis of iPSCs is required for mesoderm conversion and consequent EMT of

177 the surviving iPSC population.

178

179

\section{ATP provides an essential signal through the purinergic P2Y receptor}

180 Apoptotic cells generate soluble find-me signals that recruit macrophages and membrane-

181 associated eat-me signals to promote engulfment ${ }^{17,18}$. Nucleotides (ATP, UTP) have been

182 identified as potent find-me signals ${ }^{19}$. We first tested, using a luciferase assay, whether

183 apoptosing iPSCs release ATP. Treatment with CHIR caused a significant increase in

184 extracellular ATP within $8 \mathrm{hrs}$ (Fig 5D). Moreover, treatment with apyrase, to hydrolyze

185 nucleotides, partially blunted the EMT induced by CM on BAX/BAK DKO (Fig. 5E-F). This result was confirmed in a setting where apoptosis was blocked in WT cells using Q-VD-OPH for 24hrs prior to adding the $\mathrm{CM}+/$ - apyrase (Supp. Fig S5B-C). Moreover, WT hiPSCs co-treated with CHIR and apyrase showed a two-fold reduction in Snail and Slug expression (Fig 5G, H). Purinergic P2Y receptors bind ATP among other nucleotides, and function as chemo-attractants for macrophages ${ }^{19,20}$. Strikingly, the P2 receptor inhibitor suramin totally blocked EMT in WT iPSCs treated with CHIR, even though many of these cells still underwent apoptosis (Fig 5I). Snail and Slug were also completely suppressed (Fig. 5J-K). Additionally, suramin blocked the effect of $\mathrm{CM}$ on $B A K / B A X$ DKO cells, preventing EMT and accompanying gene expression changes (Fig 5L-M). Finally, addition of ATP to BAK/BAX DKO cells treated with CHIR also induced a partial EMT (Fig 5N-P). However, induction of apoptosis by UV irradiation, in the absence of WNT signaling, did not cause EMT (Supp. Fig. S5D-E). We conclude, therefore, that WNT signaling induces two distinct and complementary responses in pluripotent stem cells, both of which are needed for commitment to cardiogenesis: the first activating early apoptosis, triggering the release of nucleotides, including ATP, which through P2Y receptor engagement act in a paracrine fashion to permit the stem cells to enter the mesoderm lineage; while the second drives differentiation of the responsive cells through 


\section{Discussion}

207 Apoptotic cells release find-me and eat-me signals that ensure their rapid clearance from

208 tissues by macrophages and other phagocytic cells ${ }^{7,18}$. Find-me signals include several

209 molecules, including nucleotides that are recognized by purinergic G-protein coupled P2Y

210 receptors and act as chemo-attractants ${ }^{9}$. P2Y agonists have multiple biological functions in

211 addition to apoptotic clearance ${ }^{20}$ but have not previously been implicated in early developmental

212 decisions. Our discovery that suppression of apoptosis in human iPSCs and ESCs completely

213 blocks specification along the mesoderm lineage in response to WNT activation was, therefore,

214 highly unexpected. Even early changes in gene expression, such as the induction of EOMES,

215 are prevented, resulting in a later block in SNA/1/2 expression and in the subsequent EMT,

216 which we showed is essential for cardiac mesoderm commitment. This blockade must, however,

217 occur after escape from pluripotency, because the drop in Nanog expression induced by WNT

218 activation occurred normally. Moreover, BAKJBAX double KO iPSCs can still successfully enter

219 the neural tube lineage ${ }^{15}$. A previous report identified caspase activity as being required for the

220 differentiation of ESCs in response to retinoic acid ${ }^{13}$. However, the mechanism in this case

221 appears to be through caspase-induced cleavage and deactivation of Nanog, rather than

222 through the generation of a soluble paracrine signal. Apoptosis occurs pre-gastrulation during

223 mouse embryogenesis ${ }^{16}$, but apoptosis-defective mice generally progress through

224 embryogenesis. CASP3 /CASP7 double KO mice die perinatally from cardiovascular defects ${ }^{21}$.

225 A triple KO mouse line lacking BAX, BAK and BOK also develops through embryogenesis ${ }^{22}$.

226 This observation emphasizes the idea that other types of cell death (such as ferroptosis) might

227 also occur in embryogenesis to release nucleotides; additionally, nucleotides can be released

228 through pannexin 1 channels in response to multiple other stresses, which might provide the

229 necessary signal in embryogenesis ${ }^{20,23}$. Finally, we noted that ATP was insufficient to trigger

$230100 \%$ of the cells to undergo EMT; moreover, the conversion occurs in clusters across the cell

231 colonies. It is possible that additional metabolites released by apoptosing cells contribute to the

232 signal, or that the nucleotides are degraded before they can trigger differentiation of the entire

233 cell population. The patchiness of the response suggests cell-cell communication might promote

234 the EMT, or that groups of cells are in different initial states that are less or more susceptible to

235 purinergic signaling.

236 Overall, it is remarkable that the death of a fraction of pluripotent stem cells is required for

237 differentiation of the survivors, through a paracrine find-me signal that usually functions for

238 apoptotic cell clearance. It will be of interest to determine if other developmental processes

239 require similar signaling mechanisms. 
Material \& Methods

244 Reagents

245 Common lab reagents are listed in Table 1.

\section{Cell lines and Cell culture}

GM25256 hiPSCs were obtained from the Coriell Institute and were derived from a healthy 30 year-old male. mEGFP-TJP1 knock-in GM25256 hiPSC cell line was obtained from the Allen Cell Collection, Coriell Institute (Cell ID AICS-0023 cl.20). BAX/BAK double knock-out hiPSC GM25256 cell line were obtained from Dr. Vivian Gama (Joshi et al, https://doi.org/10.1038/s41419-02003002-x). Human embryonic stem cell line H9 (WA09) was obtained from WiCell Research

253 Institute (Wisconsin). All experiments using hESCs were performed using the WA09 (H9) cell

254 line under the supervision of the Vanderbilt Institutional Human Pluripotent Cell Research

255 Oversight (VIHPCRO) Committee (Protocol IRB \# 160146 to VG).

256 hiPSCs and hESCs H9 cell lines were cultured on Matrigel coated 6-well plates (Matrigel diluted 257 at $42 \mu \mathrm{g} / \mathrm{mL}$ in DMEM/F12 media) and grown in mTeSR1 medium. Medium was changed daily 258 until cells reached 70\% confluence. Cells were passaged using Gentle cell dissociation reagent 259 for 4 min, resuspended in mTseR1 as small clusters and replated at 1:7.

260 HEK-293T cells were obtained from ATCC and maintained in Dulbecco's modified Eagle's

261 medium supplemented with $10 \%$ fetal bovine serum and passaged at 1:10 every 2-3 $d$.

262 All cell lines used in this study were maintained at $37{ }^{\circ} \mathrm{C}$ and under $5 \% \mathrm{CO}_{2}$.

\section{Cell freezing and thawing}

265 hiPSCs and hESCs were collected as previously described and centrifuged at $1000 \mathrm{rpm}$ for $3 \mathrm{~min}$.

266 Pellets were resuspended in mTeSR1 supplemented with 10\% DMSO and aliquoted in cryovials.

267 Cells were first transferred at $-80^{\circ} \mathrm{C}$ for $24 \mathrm{~h}$ before long-term storage in liquid nitrogen.

268 hiPSCs and hESCs were slowly thawed using mTeSR1 media, centrifuged and resuspended in 269 mTeSR1 supplemented with $10 \mu \mathrm{M}$ of $\mathrm{Y}-27632$ for $24 \mathrm{~h}$. 


\section{Cardiomyocyte differentiation protocol}

272 This protocol was adapted from Lian et al ${ }^{2,24}$ (GiWi protocol).

273 Briefly once confluency reached 70-80\%, cells were treated with RPMI 1640 supplemented with

2741 1X B27 minus Insulin and $7.5 \mu \mathrm{M}$ CHIR-99021 for 48h. At 48h after CHIR addition, media was

275 aspirated and replaced with RPMI 1640, supplemented with 1X B27 minus Insulin and $7.5 \mu \mathrm{M}$ of

276 IWP-2 for 48h. Then, cells were incubated for 48h with RPMI 1640/1X B27 minus Insulin, before

277 maintaining them in RPMI 1640/1X B27 plus insulin every $3 \mathrm{~d}$. Spontaneous and homogenous

278 beating should be observed within 10-12 days after the protocol initiation.

279 HiPSC-derived cardiomyocytes (hiPSC-CMs) used in Figure 5 G were generated using the small

280 molecules CHIR-99021 (Selleck Chemicals) and IWR-1 (Sigma). Cardiac differentiation media

281 were defined as M1 (RPMI 1640 with glucose with B27 minus insulin), M2 (RPMI 1640 minus

282 glucose with B27 minus insulin), and M3 (RPMI 1640 with glucose with B27). When hiPSCs

283 reached $60 \%$ confluence, cardiac differentiation was initiated (day 0 ). At d 0, hiPSCs were

284 supplemented in M1 with $6 \mu \mathrm{M}$ CHIR-99021. On d 2, the media was changed to M1. On d 3,

285 cells were treated with $5 \mu \mathrm{M}$ IWR-1 in M1. Metabolic selection was started at day 10 and cells

286 were treated with M2 from d 10 to 16 . On d 16, cells were transitioned to M3. Media was

287 changed every other day until d 30.

\section{SDS-PAGE and Western blotting}

290 Cells were washed in 1X PBS. Lysates were obtained by scraping cells in lysis buffer (150 mM

$291 \mathrm{NaCl}, 10 \mathrm{mM}$ Tris-HCl pH 7.5, $1 \mathrm{mM}$ ethylenediaminetetraacetic acid (EDTA), 1\% Triton X-100,

$2920.1 \%$ SDS, $1 \mathrm{X}$ protease and phosphatase inhibitors) followed by a 5 min incubation on ice and

293 centrifugation at $16000 \mathrm{rpm}$ for $10 \mathrm{~min}$ at $4^{\circ} \mathrm{C}$. Protein concentration was measuring using

294 Precision Red.

$29530 \mu \mathrm{g}$ of proteins were resolved on bis-tris acrylamide gels and transferred onto nitrocellulose 296 membrane for $90 \mathrm{~min}$ at 110V. Membranes were blocked for $30 \mathrm{~min}$ in $5 \%$ non-fat milk in TBS-T

297 (10 mM Tris $\mathrm{pH}$ 8.0, $150 \mathrm{mM} \mathrm{NaCl}, 0.5 \%$ Tween 20) before overnight incubation with primary 298 antibodies (Table 2 ) at $4^{\circ} \mathrm{C}$ with gentle rocking. Membranes were washed in TBS-T and 
299

300

incubated $1 \mathrm{~h}$ at room temperature with Alexa-Fluor conjugated secondary antibodies (Table 2). Membranes were washed in TBS-T and scanned using the LI-COR Odyssey CLx. All images were analyzed using Image Studio Lite v. 5.2.5.

\section{Immunofluorescence}

Cells were grown on Matrigel-coated coverslip, fixed with 4\% paraformaldehyde for 10 min, permeabilized (20 mM glycine, $0.05 \%$ Triton X-100) for 10 min and blocked with 5\% BSA-PBS for $30 \mathrm{~min}$. Primary and secondary antibodies were diluted in blocking buffer and incubated for $1 \mathrm{~h}$ in a dark, humidified chamber. Coverslips were washed three times in PBS before being mounted on glass slides using Fluoromount-G ${ }^{\mathrm{TM}}$ Slide Mounting Medium. Images were taken using an inverted Nikon A1-R confocal microscope equipped with a 40x oil objective (NA 1.2). $0.5 \mu \mathrm{m}$ Z-stack covering the entire cell height were obtained.

Super-resolution images for Figure 5G were acquired using a Nikon SIM microscope equipped with a 1.49 NA 100x Oil objective an Andor DU-897 EMCCD camera. Images were processed and analyzed using Fiji software (ImageJ version 2.1.0/1.53c).

\section{Live Cell Imaging}

mEGFP-TJP1 hiPSCs were plated on Matrigel-coated MaTek 35mm dishes. Cells were imaged every 10-15 min on a Nikon A1-R with a 40X oil objective (NA 1.2) and equipped with a heated CO2 chamber. 2-3 $\mu \mathrm{m}$ Z-stack were obtained and images were processed and analyzed using Fiji software (ImageJ version 2.1.0/1.53c).

\section{Generation of knock-out cell lines.}

Single-guide RNA was selected using ChopChop ${ }^{25}$ and Benchling design tools and are listed in Table 3. Annealed oligonucleotides were cloned into pLentiCrispRv2-Puro as described by Sanjana et al (10.1038/nmeth.3047). HEK-293T cells were seeded on $15 \mathrm{~cm}$ dish to 50\% confluence and transfected using calcium phosphate. Briefly, $50 \mu \mathrm{g}$ of the lentiviral plasmid, $37.5 \mu \mathrm{g}$ of pSPAX2 (Addgene 8454) and $15 \mu \mathrm{g}$ of pMD2G (Addgene 12260) were combined to $1125 \mu \mathrm{l}$ of sterile water, complemented with $125 \mu \mathrm{l}$ of $2.5 \mathrm{M} \mathrm{CaCl}_{2}$. While vortexing, $1.25 \mathrm{ml}$ of 
328 filter sterilized 2x HEPES-buffered saline (50 mM Hepes, $10 \mathrm{mM} \mathrm{KCl,} 12 \mathrm{mM}$ Dextrose, $280 \mathrm{mM}$

$329 \mathrm{NaCl}, 1.5 \mathrm{mM} \mathrm{Na}_{2} \mathrm{PO}_{4}, \mathrm{pH} 7.04$ ) was added, and the solution was incubated 5 min at RT before

330 adding to HEK-293T cells. Medium was removed after $6-8 \mathrm{~h}$ and replaced with $15 \mathrm{ml}$ of $10 \%$ FBS

331 DMEM. Lentiviruses were collected after 48h, concentrated using Amicon centrifugal filter units

332 (100 kDa cut-off) and stored at $-80^{\circ} \mathrm{C}$. hiPSCs were transduced in suspension for $24 \mathrm{~h}$ and then

333 selected using $1 \mu \mathrm{g} / \mathrm{mL}$ Puromycin.

\section{RNA isolation and RT-qPCR}

336 RNA was isolated using RNeasy Mini kit. $1 \mu \mathrm{g}$ of RNA was reverse transcribed to cDNA using

337 SuperScript III First-Strand Synthesis System and diluted 1:10 in water. $4.5 \mu \mathrm{L}$ of cDNA was 338 mixed with $7.5 \mu \mathrm{L}$ Maxima SYBR Green/Fluorescein Master Mix and $3 \mu \mathrm{L}$ of primers (1 mM 339 each) (Table 4). qPCR was performed on a BioRad CFX96 Thermocycler and Ct values from 340 technical triplicates were average and used to calculate the relative gene expression normalized 341 to GAPDH, using the $\Delta \Delta \mathrm{Ct}$ formula.

\section{Annexin V-APC assay}

344 Protocol was adapted from the Annexin V apoptosis kit APC. Briefly, cells were collected as 345 single cell suspension by incubation in gentle cell dissociation buffer for $8 \mathrm{~min}$ at $37^{\circ} \mathrm{C}$. Pellet 346 was washed in once in PBS and once in $1 \mathrm{X}$ binding buffer. Cells were resuspended in $100 \mu \mathrm{L} 1 \mathrm{X}$ 347 binding buffer and incubated $15 \mathrm{~min}$ at room temperature with $5 \mu \mathrm{L}$ of Annexin-APC. Cells were 348 washed in $1 \mathrm{X}$ binding buffer, resuspended in $200 \mu \mathrm{L}$ of binding buffer and incubated with $5 \mu \mathrm{L}$ 349 of propidium iodide. Cells were passed through a $70 \mu \mathrm{m}$ strainer prior to cytometry analysis 350 using a 3-laser Fortessa flow cytometer.

352 ATP release - Luciferase assay

353 hiPSCs were treated with CHIR-99021 as described previously plus DMSO or $10 \mu \mathrm{M}$ Q-VD-OPH. 354 Aliquots of culture medium (300 $\mu \mathrm{l})$ were taken at indicated timepoints and mixed with $100 \mu \mathrm{L}$ 355 of 4 X RealTime-Glo extracellular ATP assay reagent (Promega) reconstituted in RPMI 356 1640/B27(-Ins) medium. Technical triplicates of $100 \mu \mathrm{L}$ were dispensed into a dark edged glass- 
357 bottom 96-well plate. Luminescence was measured after 30min using a HT-Synergy plate

358 reader. Luminescence was subtracted for background.

359 A standard curve was obtained by serial dilution of ATP in RPMI 1640/B27(-Ins) media followed

360 by the luciferase assay as described above. Simple linear regression was applied to transform

361 luminescence values to ATP concentration.

\section{Statistical analysis}

364 Datasets were analyzed using Prism8 (v.8.4.3) and tested for normality prior to applying the 365 appropriate statistical test, as mentioned in each figure legend. Error bars represent S.D unless 366 stated otherwise. Significance levels are given as follows: n.s. (not significant) : P > 0.05, ${ }^{*} \mathrm{P} \leq$ $0.05, * * \mathrm{P} \leq 0.01, * * * \mathrm{P} \leq 0.001, * * * * \mathrm{P} \leq 0.0001$.

368 All experiments were repeated at least three times independently as biological repeats unless 369 stated otherwise.

370 Datasets are color-coded to reflect the variability between biological repeats.

371

\section{Acknowledgments}

373 We thank Piyush Joshi for establishing the BAX/BAK DKO hiPSCs and Megan Rasmussen for the 374 providing the SIM pictures of hiPSC-derived cardiomyocytes. We thank members of the Macara 375 lab for discussion. This work was supported by GM070902 from NIGMS, CA197571 from the NCI 376 (both to I.G.M) and by 1R35GM128915-01 (to V.G.).

\section{Contributions}

379 Conceptualization, L.F., V.G. and I.G.M. ; Methodology, L.F., I.G.M. ; L.F. performed experiments 380 and analyzed data. V.G. provided resources. L.F. prepared the figures. I.G.M and LF. wrote and 381 edited the manuscript. I.G.M. supervised the work.

\section{Declaration of interests}

384 The authors declare no competing interests. 


\section{References}

1 Ueno, S. et al. Biphasic role for Wnt/beta-catenin signaling in cardiac specification in zebrafish and embryonic stem cells. Proc Natl Acad Sci U S A 104, 9685-9690, doi:10.1073/pnas.0702859104 (2007).

2 Lian, X. et al. Robust cardiomyocyte differentiation from human pluripotent stem cells via temporal modulation of canonical Wnt signaling. Proc Natl Acad Sci U S A 109, E1848-1857, doi:10.1073/pnas.1200250109 (2012).

3 Rao, J. et al. Stepwise Clearance of Repressive Roadblocks Drives Cardiac Induction in Human ESCs. Cell Stem Cell 18, 554-556, doi:10.1016/j.stem.2016.03.008 (2016).

4 Loh, K. M. et al. Mapping the Pairwise Choices Leading from Pluripotency to Human Bone, Heart, and Other Mesoderm Cell Types. Cell 166, 451-467, doi:10.1016/j.cell.2016.06.011 (2016).

5 Bedzhov, l. et al. Adhesion, but not a specific cadherin code, is indispensable for ES cell and induced pluripotency. Stem Cell Res 11, 1250-1263, doi:10.1016/j.scr.2013.08.009 (2013).

6 Takahashi, K. \& Yamanaka, S. Induction of pluripotent stem cells from mouse embryonic and adult fibroblast cultures by defined factors. Cell 126, 663-676, doi:10.1016/j.cell.2006.07.024 (2006).

7 Liu, X. et al. Sequential introduction of reprogramming factors reveals a time-sensitive requirement for individual factors and a sequential EMT-MET mechanism for optimal reprogramming. Nat Cell Biol 15, 829-838, doi:10.1038/ncb2765 (2013).

8 Li, R. et al. A mesenchymal-to-epithelial transition initiates and is required for the nuclear reprogramming of mouse fibroblasts. Cell Stem Cell 7, 51-63, doi:10.1016/j.stem.2010.04.014 (2010).

9 Samavarchi-Tehrani, P. et al. Functional genomics reveals a BMP-driven mesenchymalto-epithelial transition in the initiation of somatic cell reprogramming. Cell Stem Cell 7, 64-77, doi:10.1016/j.stem.2010.04.015 (2010).

10 Nowotschin, S. \& Hadjantonakis, A. K. Guts and gastrulation: Emergence and convergence of endoderm in the mouse embryo. Curr Top Dev Biol 136, 429-454, doi:10.1016/bs.ctdb.2019.11.012 (2020).

11 D'Amour, K. A. et al. Efficient differentiation of human embryonic stem cells to definitive endoderm. Nat Biotechnol 23, 1534-1541, doi:10.1038/nbt1163 (2005).

$12 \mathrm{Li}$, Q. et al. A sequential EMT-MET mechanism drives the differentiation of human embryonic stem cells towards hepatocytes. Nat Commun 8, 15166, doi:10.1038/ncomms15166 (2017).

13 Fujita, J. et al. Caspase activity mediates the differentiation of embryonic stem cells. Cell Stem Cell 2, 595-601, doi:10.1016/j.stem.2008.04.001 (2008).

14 Bondue, A. et al. Defining the earliest step of cardiovascular progenitor specification during embryonic stem cell differentiation. J Cell Biol 192, 751-765, doi:10.1083/jcb.201007063 (2011). 
$42815 \quad$ Joshi, P. et al. Modeling the function of BAX and BAK in early human brain development using iPSC-derived systems. Cell Death Dis 11, 808, doi:10.1038/s41419-020-03002-x (2020).

43116 Manova, K. et al. Apoptosis in mouse embryos: elevated levels in pregastrulae and in the distal anterior region of gastrulae of normal and mutant mice. Dev Dyn 213, 293-308, doi:10.1002/(SICI)1097-0177(199811)213:3<293::AID-AJA6>3.0.CO;2-D (1998). Ravichandran, K. S. Beginnings of a good apoptotic meal: the find-me and eat-me signaling pathways. Immunity 35, 445-455, doi:10.1016/j.immuni.2011.09.004 (2011). Lauber, K., Blumenthal, S. G., Waibel, M. \& Wesselborg, S. Clearance of apoptotic cells: getting rid of the corpses. Mol Cell 14, 277-287, doi:10.1016/s1097-2765(04)00237-0 (2004).

Elliott, M. R. et al. Nucleotides released by apoptotic cells act as a find-me signal to promote phagocytic clearance. Nature 461, 282-286, doi:10.1038/nature08296 (2009). Erb, L. \& Weisman, G. A. Coupling of P2Y receptors to $G$ proteins and other signaling

$44421 \quad$ Lakhani, S. A. et al. Caspases 3 and 7: key mediators of mitochondrial events of apoptosis. Science 311, 847-851, doi:10.1126/science.1115035 (2006). Ke, F. F. S. et al. Embryogenesis and Adult Life in the Absence of Intrinsic Apoptosis Effectors BAX, BAK, and BOK. Cell 173, 1217-1230 e1217, doi:10.1016/j.cell.2018.04.036 (2018). conduits for ATP. FEBS Lett 572, 65-68, doi:10.1016/j.febslet.2004.07.009 (2004).

24 Lian, X. et al. Directed cardiomyocyte differentiation from human pluripotent stem cells by modulating Wnt/beta-catenin signaling under fully defined conditions. Nat Protoc $\mathbf{8}$, 162-175, doi:10.1038/nprot.2012.150 (2013). editing. Nucleic Acids Res 47, W171-W174, doi:10.1093/nar/gkz365 (2019). 
Figure 1

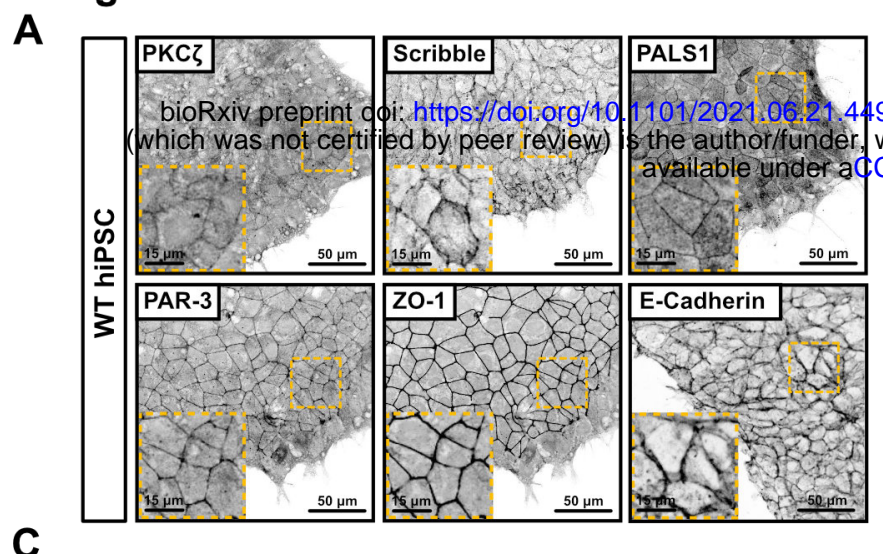

B

C
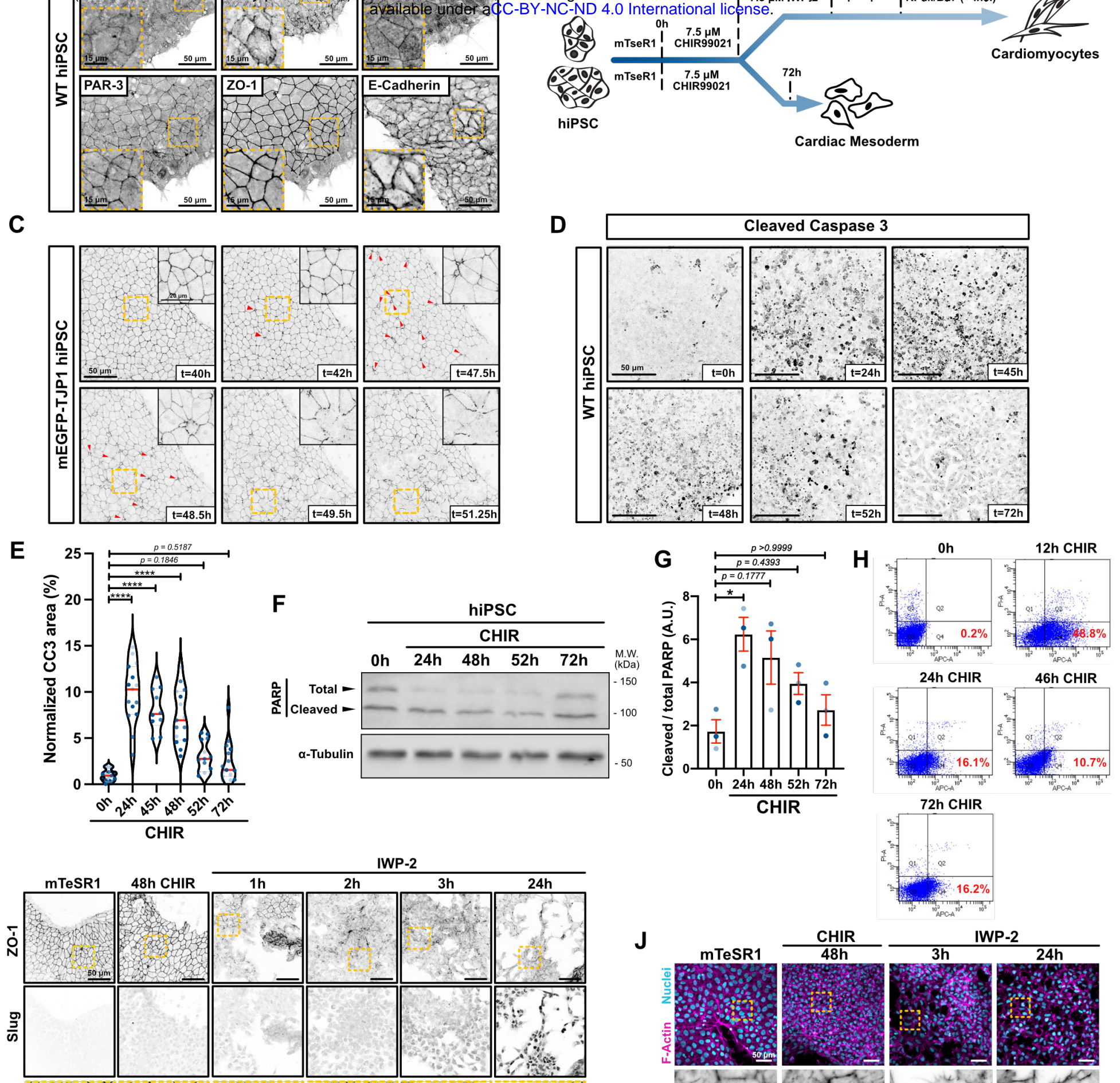

Cardiomyocytes
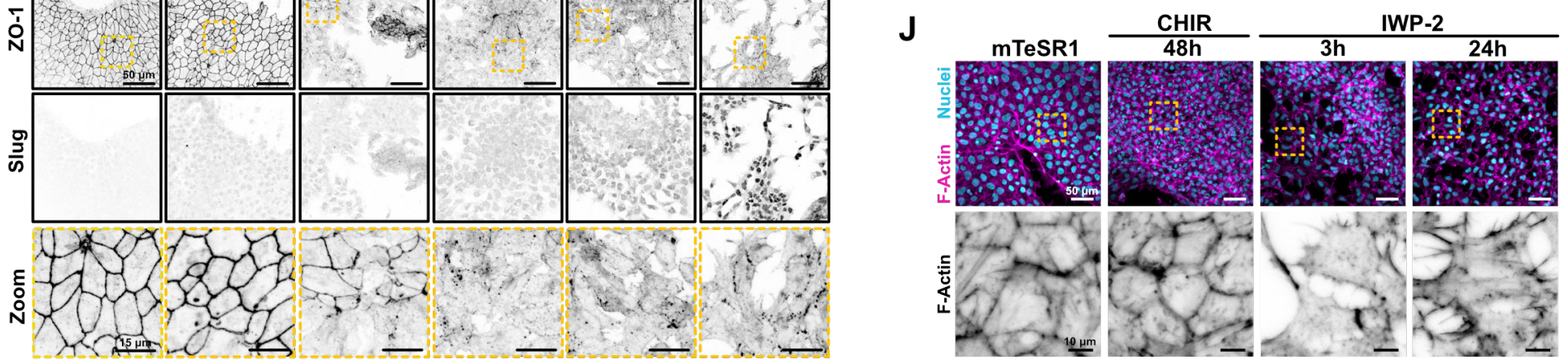

K

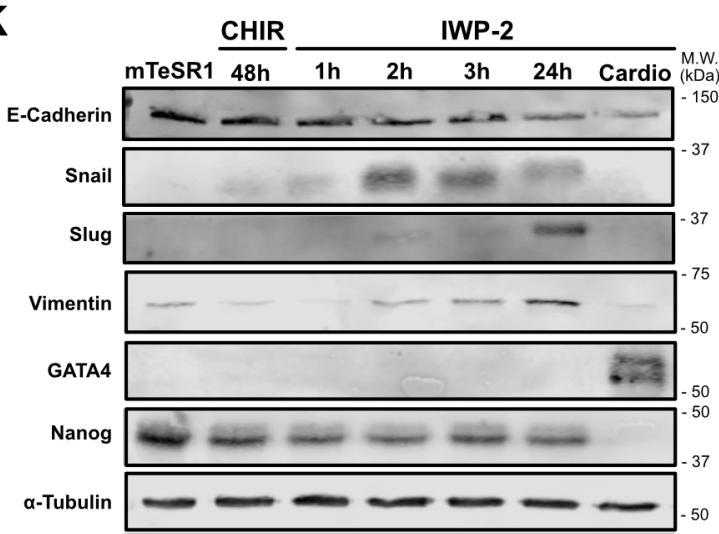

L

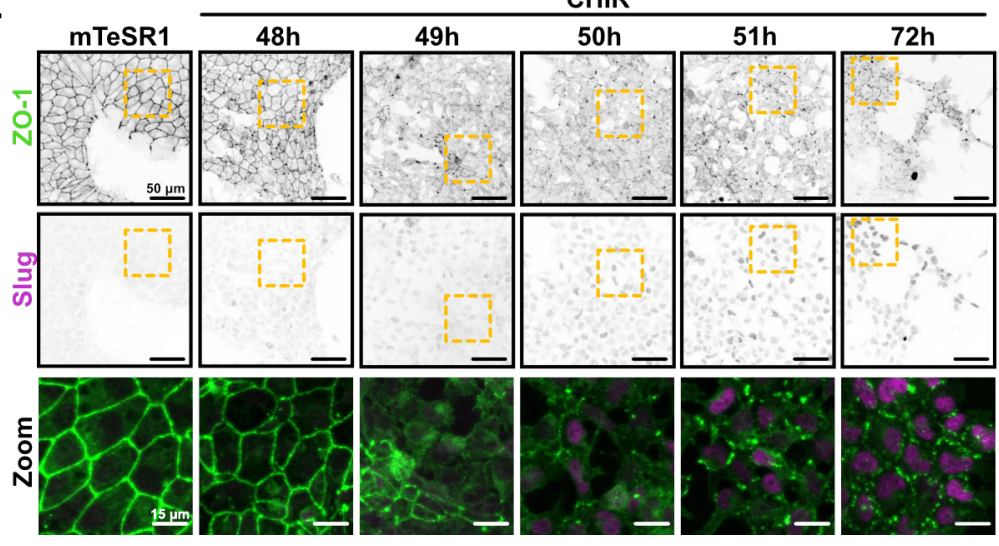




\section{Figure 1}

A) Immunofluorescence of hiPSC GM25256 stained for epithelial/polarity markers (Tight junctions: ZO-1 ; Adherent junctions: E-Cadherin ; Baso-lateral marker: Scribble ; Apical PAR complex: PKCろ, PAR-3 ; Apical Crumbs complex: PALS1). Maximum intensity projections are shown. Scale bar $=50 \mu \mathrm{m}$. Inset represents a magnified area (yellow dotted square). Scale bar $=$ $15 \mu \mathrm{m}$.

B) Schematic of GiWi differentiation protocol (top path) (conversion of hiPSC into cardiomyocytes); or alternative cardiac mesoderm with a prolonged incubation in CHIR-99021 (bottom path).

C) Stills from Supp. Movie 1. mEGFP-TJP1 knock-in hiPSCs were imaged from 40h after CHIR treatment. Red arrows represent extruding cells. Scale bar $=50 \mu \mathrm{m}$. Magnified area (yellow dotted square) is shown on the right-hand corner. Scale bar $=20 \mu \mathrm{m}$.

D-E) Representative immunofluorescence images of wildtype hiPSCs fixed at the indicated times after CHIR addition and stained for cleaved Caspase-3. Maximum intensity projections are shown. Scale bar $=50 \mu \mathrm{m}$ (D). Violin plots quantifying the area of cleaved caspase 3-positive cells normalized to the cellular area. Independent biological repeats are color-coded $(n=3,5$ random fields of view/repeat). (Median: plain red line - Quartiles: black dotted lines). Tukey's multiple comparison was applied (**** $\mathrm{P} \leq 0.0001)(\mathrm{E})$.

F-G) Immunoblot of PARP cleavage in hiPSCs during CHIR treatment. Molecular weights (M.W.) are indicated in kDa (F). PARP cleavage was quantified by densitometry across 3 independent biological repeats (color-coded). Dunn's multiple comparison was applied (*p<0.05) (G). H) Cytometry gates from an Annexin-APC assay. Cells were treated with CHIR for different times, collected, stained and analyzed for Annexin (x-axis) and PI (y-axis). Percentage of Annexin-positive/PI-negative (Gate Q4 - Early apoptosis) is reported for each condition. I) Representative immunofluorescence images of hiPSCs (mTeSR1) or cells undergoing differentiation using CHIR/IWP-2 protocol, and stained for ZO-1 and Slug. Maximum intensity projections are shown. Scale bar $=50 \mu \mathrm{m}$. Magnified areas of the ZO-1 staining (yellow dotted square) are shown in the bottom row. Scale bar $=15 \mu \mathrm{m}$. 
J) Representative immunofluorescence images of wildtype hiPSCs fixed at the indicated times along the differentiation protocol and stained for F-actin (Phalloidin - Magenta) and nuclei (Dapi). Maximum intensity projections are shown. Scale bar $=50 \mu \mathrm{m}$. Magnified area of the Factin channel (yellow dotted square) is shown. Scale bar $=10 \mu \mathrm{m}$.

K) Immunoblots for EMT markers (E-Cadherin, Snail, Slug, Vimentin) during hiPSC differentiation to early cardiac mesoderm. Membranes were also blotted for GATA4 (cardiac specific marker) and Nanog (pluripotency marker). Molecular weights (M.W.) are indicated in kDa.

L) Representative immunofluorescence images of hiPSCs (mTeSR1) or undergoing differentiation using prolonged CHIR incubation, and stained for ZO-1 and Slug. Maximum intensity projections are shown. Scale bar $=50 \mu \mathrm{m}$. Magnified area (yellow dotted square) is shown as a merge. Scale bar $=15 \mu \mathrm{m}$. 
Figure 2
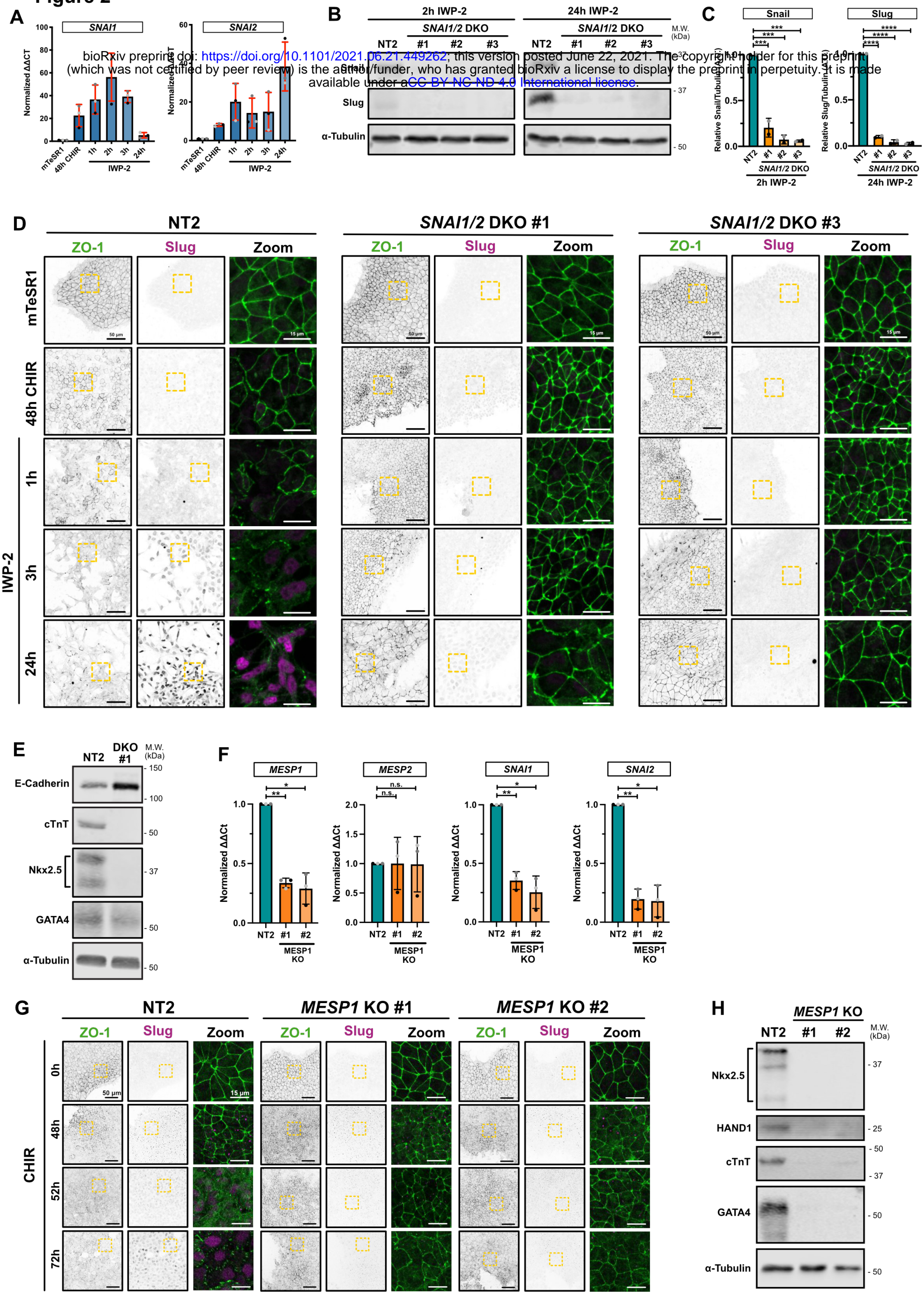


\section{Figure 2}

A) qRT-PCR showing expression of SNAI1 (Snail) and SNAI2 (Slug) during differentiation. Independent biological repeats are color-coded $(n=3)$. Error bar = Mean +/- S.D.

B-C) Immunoblot analysis of three independent SNAI1/SNAI2 double knockout (DKO) hiPSC cell lines (non-clonal). Lysates from Non-targeting (NT2) or DKO cell lines were collected $2 \mathrm{~h}$ after IWP-2 treatment (to confirm SNAI1 knockout - Left panel) or $24 \mathrm{~h}$ after IWP-2 treatment (to confirm SNAI2 knockout - Right panel). Molecular weights (M.W.) are indicated in kDa (B). Expression levels of 2 independent biological replicates were quantified by densitometry and normalized to NT2. Error bar = Mean +/-S.D. Dunnett's multiple comparison test was applied $(* * * p \leq 0.001, * * * * p \leq 0.0001)(C)$.

D) Representative immunofluorescence images of control NT2 and two independent SNAI1/SNAI2 DKO cultures, fixed at different timepoints post-induction of cardiac mesoderm induction and stained for ZO-1 (green) and Slug (Magenta). Maximum intensity projections are shown. Scale bar $=50 \mu \mathrm{m}$. Magnified area (yellow dotted square) is shown. Scale bar $=15 \mu \mathrm{m}$. E) Immunoblot of control NT2 and SNAI1/SNAI2 DKO \#1 hiPSC-derived cardiomyocytes, at 12 days post induction. Expression of EMT marker (E-Cadherin), cardiac lineage markers (cardiac Troponin T, Nkx-2.5, GATA-4) and alpha-Tubulin as loading control were analyzed. Molecular weights (M.W.) are indicated in kDa.

F) Relative gene expression of MESP1, MESP2, SNAI1 and SNAI2 obtained from control (NT2) or two independent MESP1 knockout (\#1 and \#2) were analyzed by qRT-PCR. $\triangle \triangle C$ t values from RTPCR were normalized to NT2. Independent biological repeats are color-coded $(n=3)$. Error bar $=$ Mean +/- S.D. Tukey's multiple comparison test was applied (n.s. $p>0.05, * p \leq 0.05, * * p \leq$ 0.01).

G) Representative immunofluorescence images of control NT2 and two independent MESP1 KO (\#1 and \#2) non-clonal cell lines, fixed at indicated time, and stained for ZO-1 (green) and Slug (Magenta). Maximum intensity projections are shown. Scale bar $=50 \mu \mathrm{m}$. Magnified area (yellow dotted square) is shown as a merge. Scale bar $=15 \mu \mathrm{m}$.

H) Immunoblot of control NT2 and MESP1 KO \#1 and \#2 hiPSC-derived cardiomyocytes, at 12 days post induction. Expression of cardiac lineage markers (cardiac Troponin T, Nkx-2.5, GATA- 
bioRxiv preprint doi: https://doi.org/10.1101/2021.06.21.449262; this version posted June 22, 2021. The copyright holder for this preprint (which was not certified by peer review) is the author/funder, who has granted bioRxiv a license to display the preprint in perpetuity. It is made available under aCC-BY-NC-ND 4.0 International license.

4, HAND1) and $\alpha$-Tubulin as loading control were analyzed. Molecular weights (M.W.) are indicated in kDa. 


\section{Figure 3}

A

CHIR + DMSO

CHIR + Q-VD-OPH

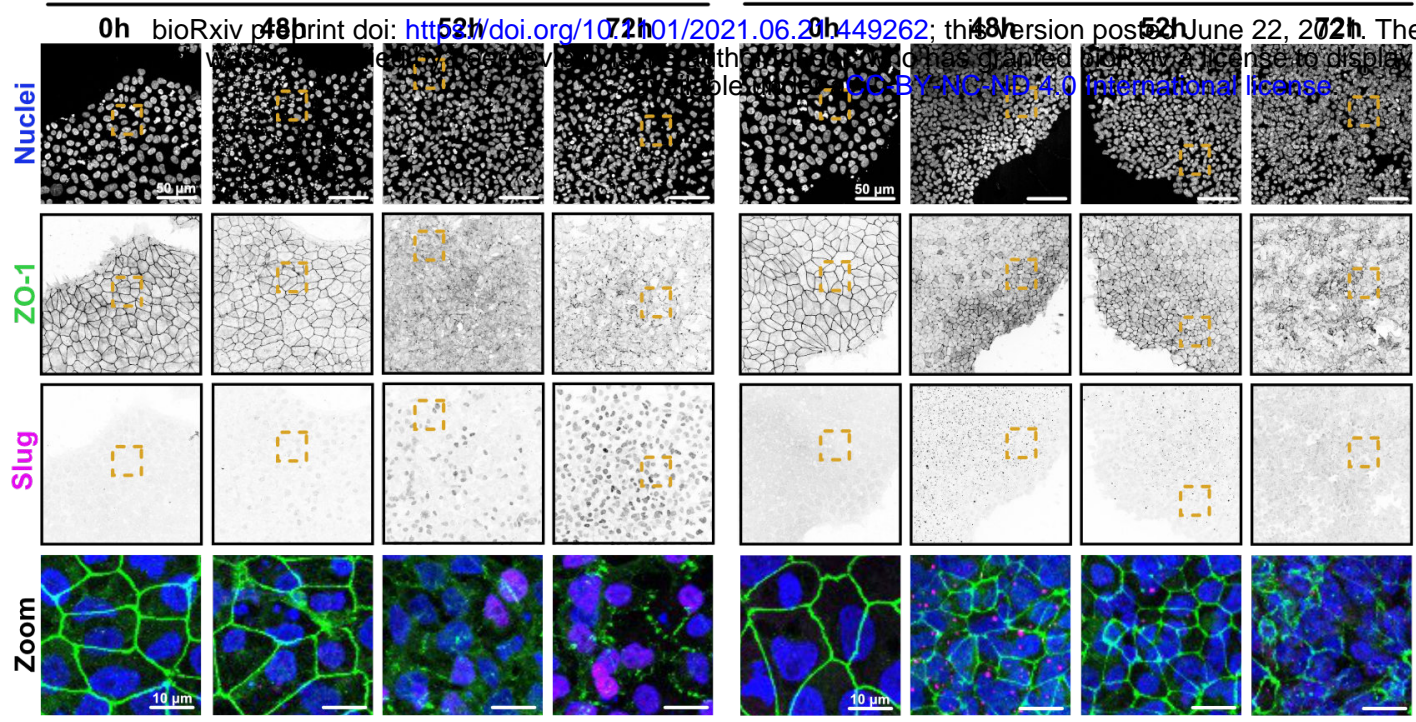

B

hiPSCs
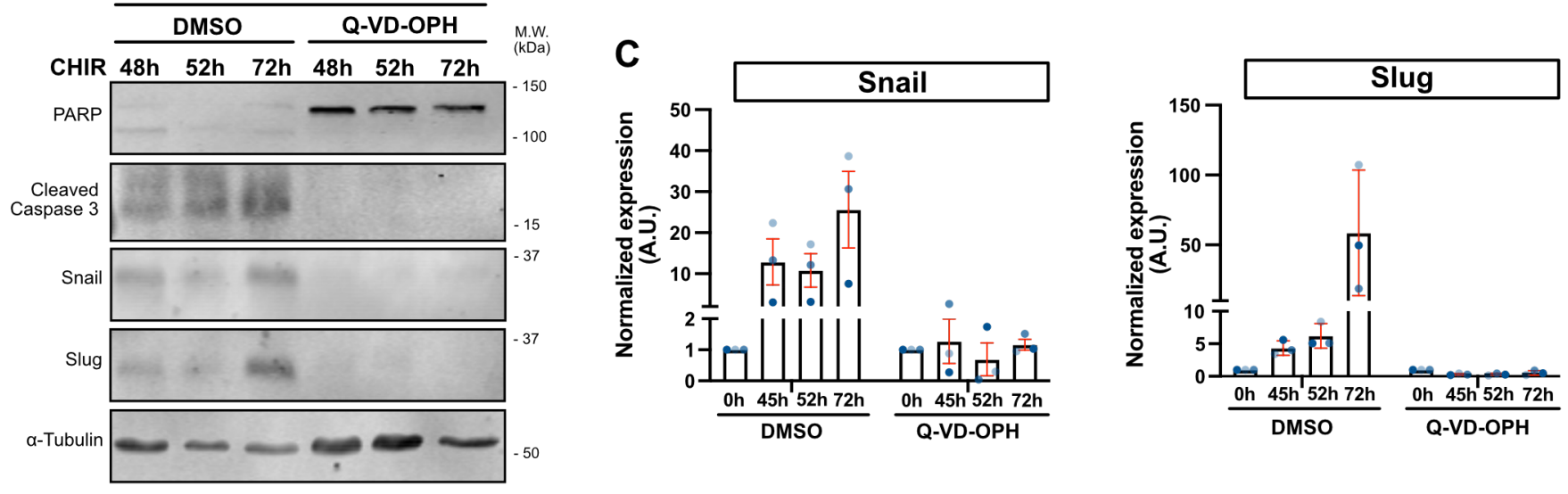

D
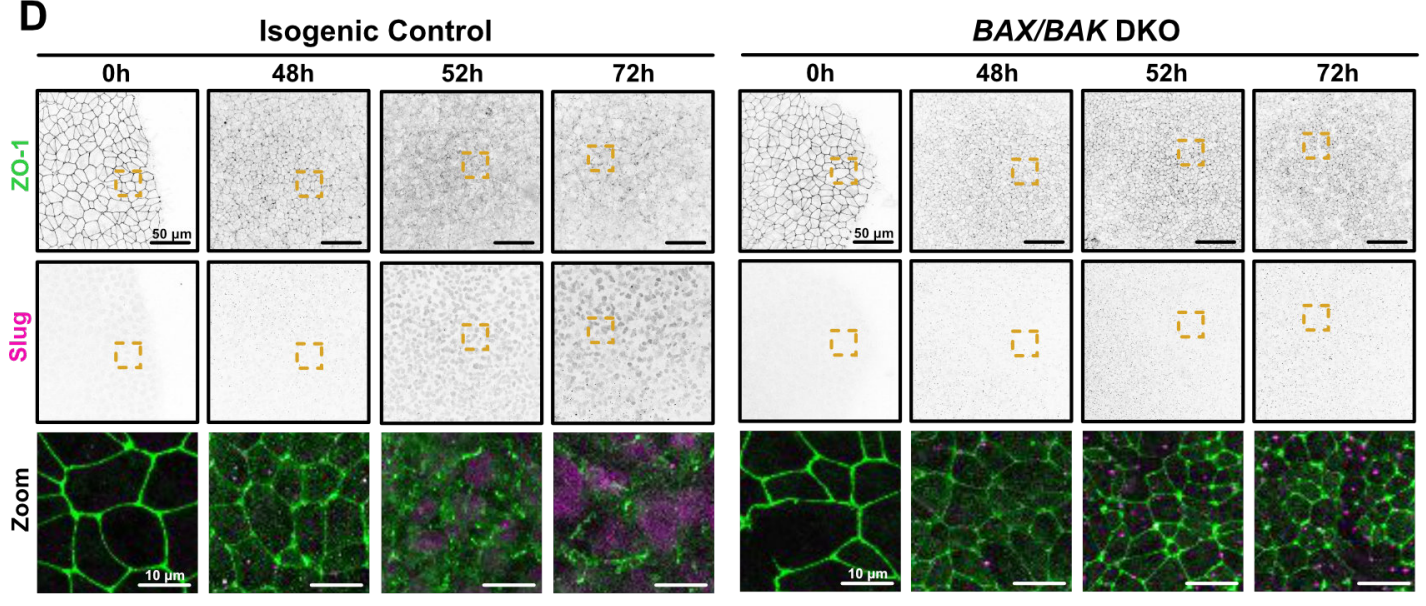

E

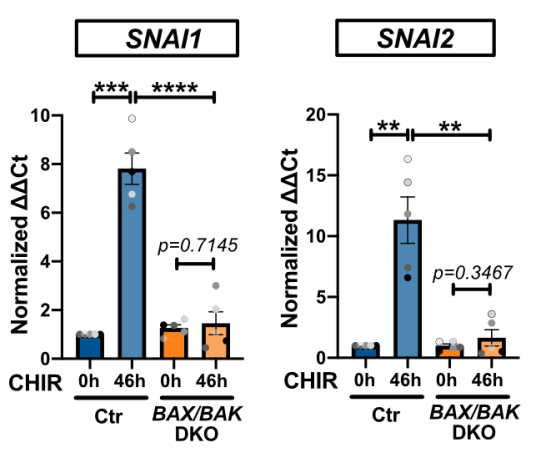

F

G

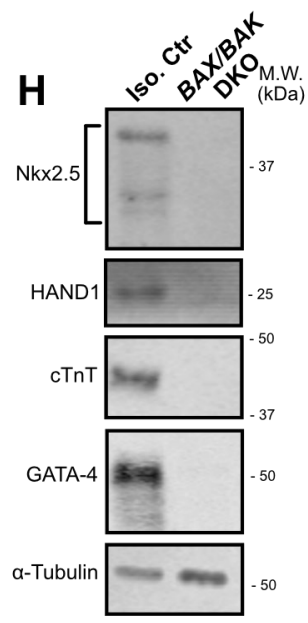

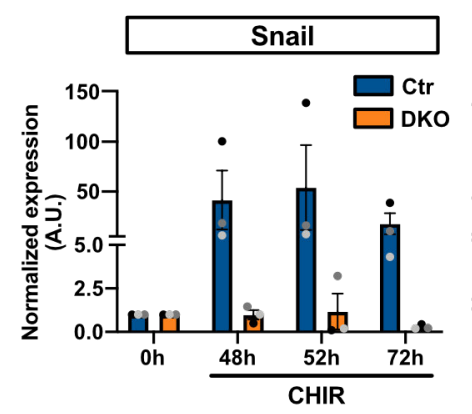

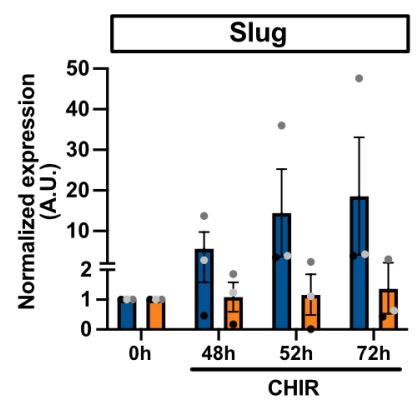




\section{Figure 3}

A) Representative immunofluorescence images of hiPSCs co-treated with CHIR and DMSO (left) or CHIR and $10 \mu \mathrm{M}$ Q-VD-OPH (right) stained for ZO-1 (green) and Slug (magenta) and nuclei (blue). Maximum intensity projections are shown. Scale bar $=50 \mu \mathrm{m}$. Magnified area (yellow dotted square) is shown as a merge. Scale bar $=10 \mu \mathrm{m}$.

B-C) Immunoblot analysis of hiPSCs co-treated with CHIR plus DMSO or $10 \mu \mathrm{M}$ Q-VD-OPH. Molecular weights (M.W.) are indicated in kDa (B). Normalized expression of Snail and Slug was quantified by densitometry across 3 independent biological replicates (color-coded). Mean +/S.E.M. (C).

D) Representative immunofluorescence images of isogenic control and $B A X / B A K$ double knockout (DKO) hiPSCs, treated with CHIR and stained for ZO-1 (green) and Slug (magenta). Maximum intensity projections are shown. Scale bar $=50 \mu \mathrm{m}$. Magnified area (yellow dotted square) is shown as a merge. Scale bar $=15 \mu \mathrm{m}$.

E) Relative gene expression of SNAI1 and SNAI2 obtained from isogenic control (Ctr) or $B A X / B A K$ DKO hiPSCs, induced or not with CHIR for $46 \mathrm{~h}$ and analyzed by qRT-PCR. $\triangle \triangle \mathrm{Ct}$ values were normalized to un-induced control cells. Independent biological repeats are color-coded $(n=5)$. Error bar = Mean +/- S.E.M. Paired t-test was applied to compare $0 \mathrm{~h}$ vs $46 \mathrm{~h}$ and unpaired t-test was applied to compare Ctr vs DKO (** $p \leq 0.01, * * * p \leq 0.001, * * * * p \leq 0.0001)$. F-G) Immunoblot analysis of isogenic control (Iso. Ctr) and BAX/BAK DKO hiPSCs, treated with CHIR. Molecular weights (M.W.) are indicated in kDa (F). Normalized expression of Snail and Slug was quantified by densitometry across 3 independent biological replicates (color-coded). Mean +/- S.E.M. (G).

H) Immunoblot of isogenic control (Iso. Ctr) and BAX/BAK DKO hiPSC-derived cardiomyocytes, at $12 \mathrm{~d}$ post induction. Expression of cardiac lineage markers (cardiac Troponin T, Nkx-2.5, GATA-4, HAND1) and $\alpha-$ Tubulin as loading control were analyzed. 


\section{Figure 4}

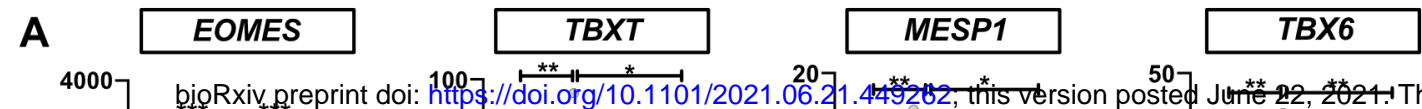

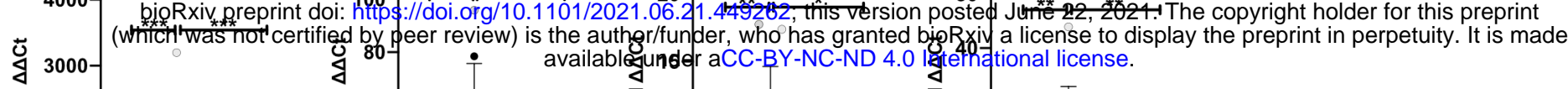

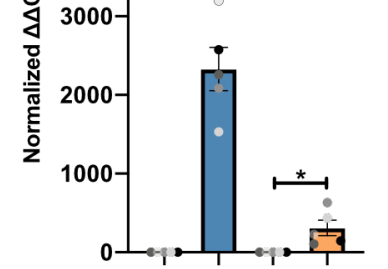

CHIR Oh 46h Oh 46h

Ctr $\frac{\substack{\text { BAX/BAK } \\ \text { DKO }}}{2}$

B
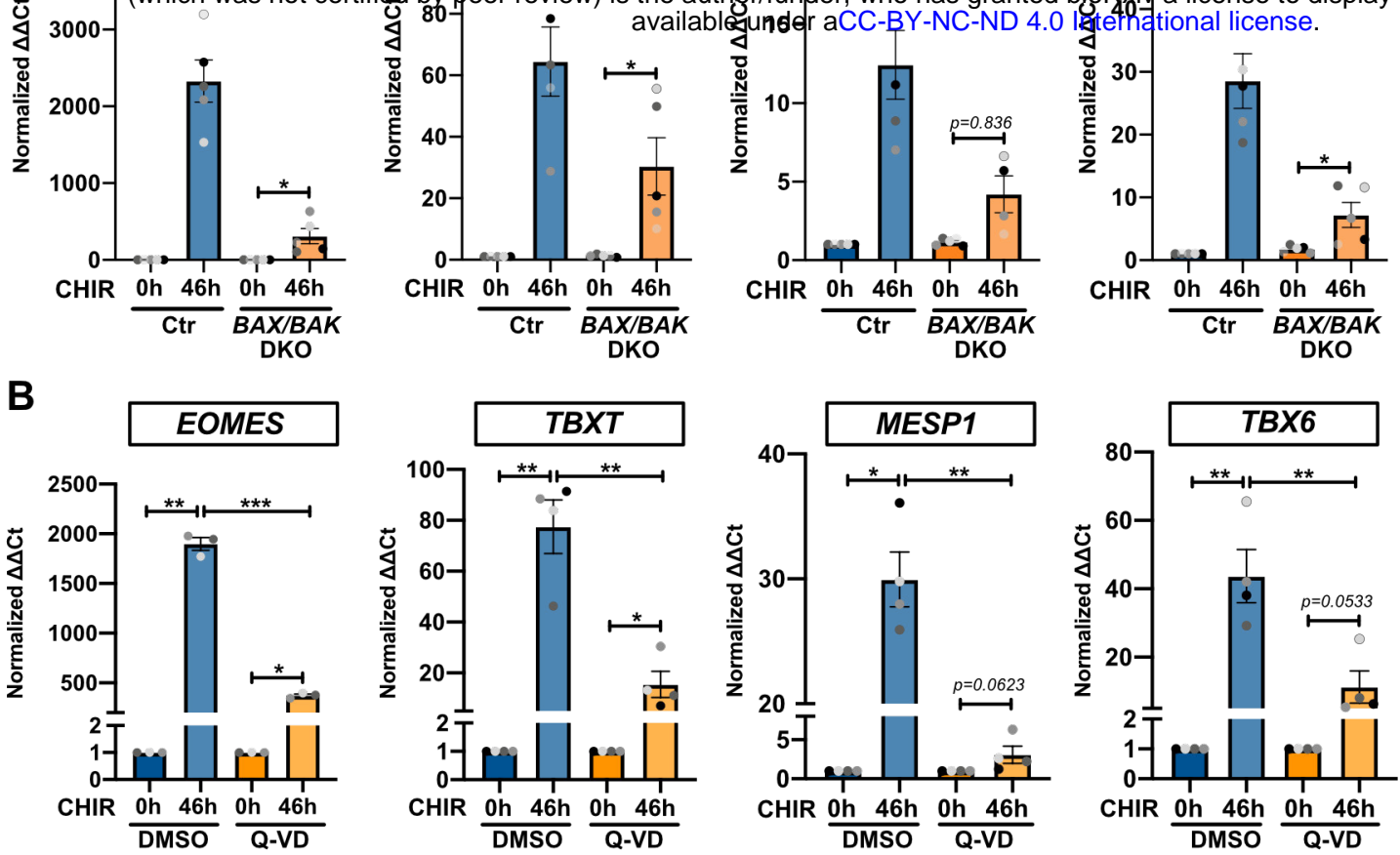

C
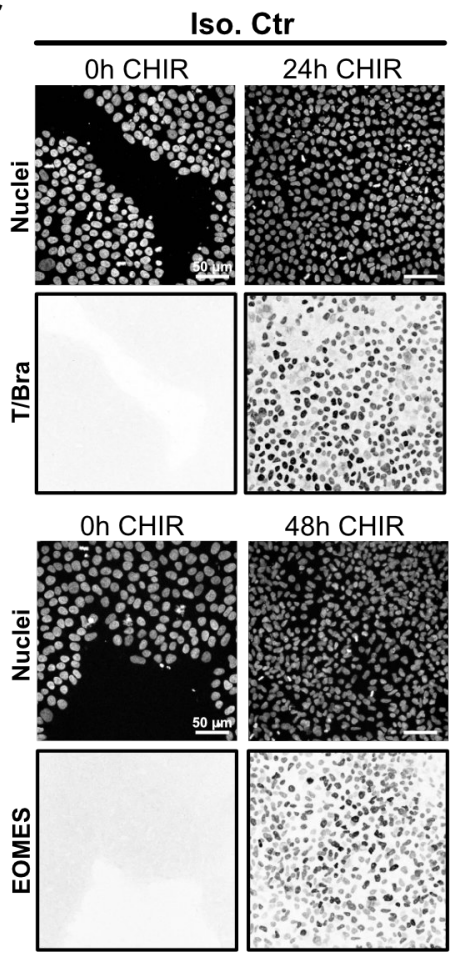

BAX/BAK DKO

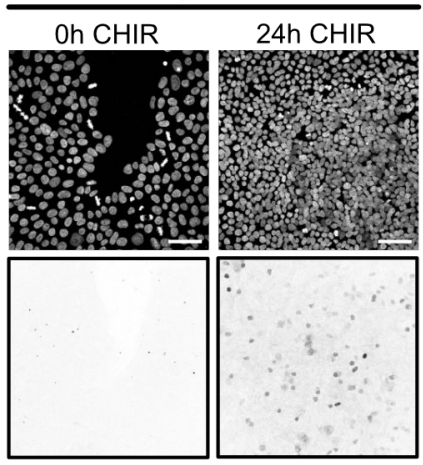

Oh CHIR

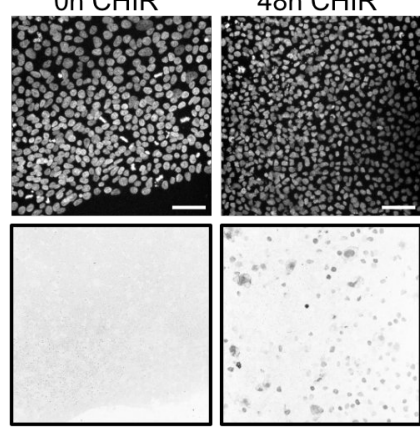

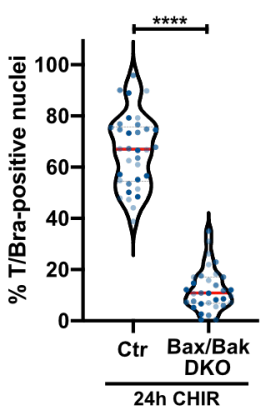

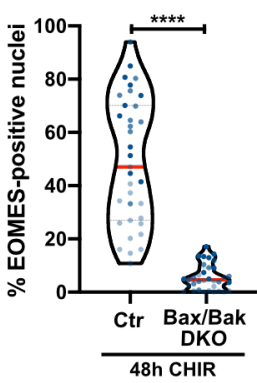

D

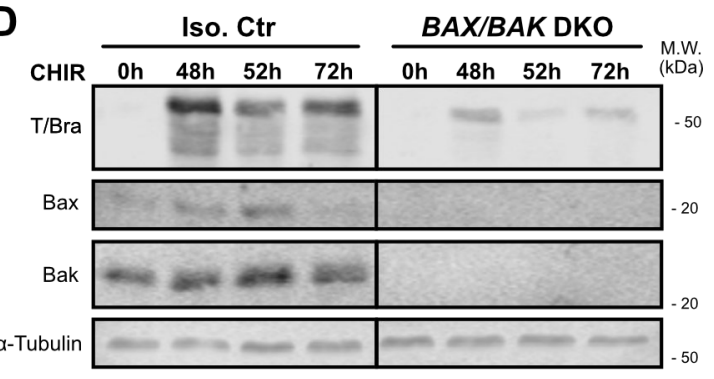

E

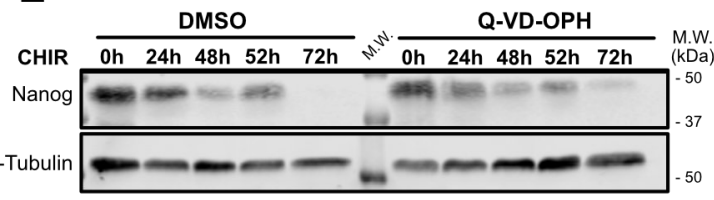

F

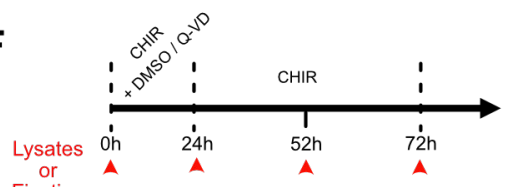

G

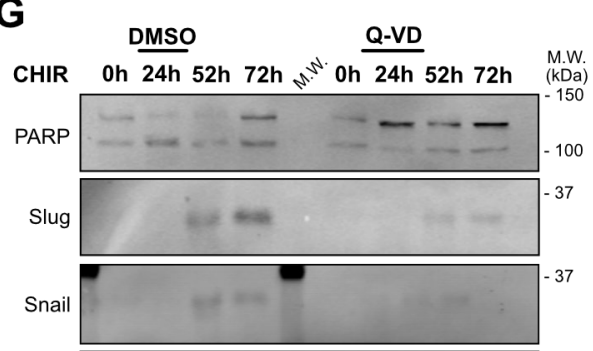

H

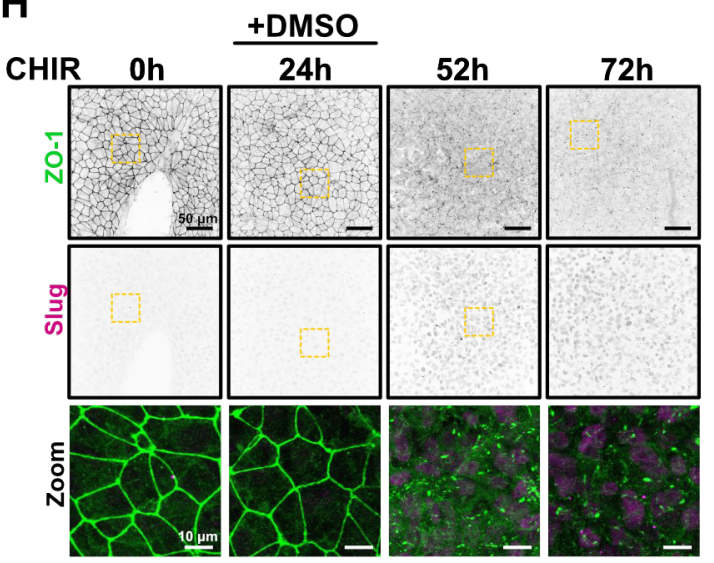

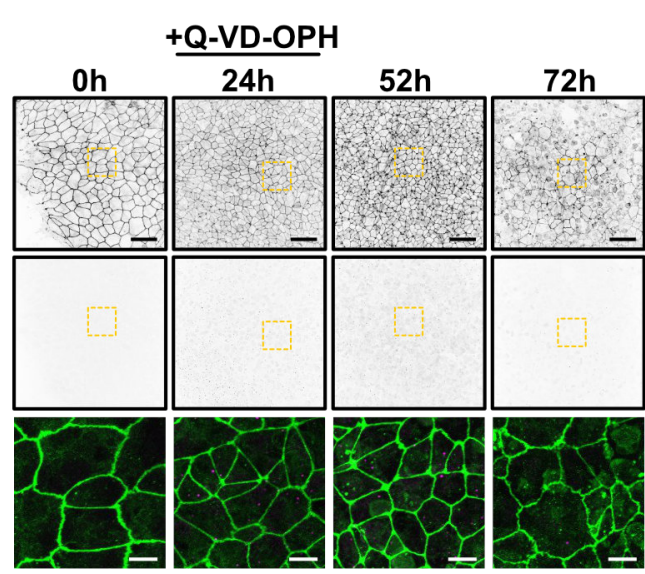




\section{Figure 4}

A) Relative gene expression of EOMES, TBXT, MESP1, TBX6 obtained from isogenic control (Ctr) or $B A X / B A K$ DKO hiPSCs, induced or not with CHIR for $46 \mathrm{~h}$ and analyzed by qRT-PCR. $\triangle \triangle \mathrm{Ct}$ values were normalized to un-induced cells. Independent biological repeats are color-coded $(n=5)$. Error bar = Mean +/- S.E.M. Paired t-test was applied to compare $0 \mathrm{~h}$ vs $46 \mathrm{~h}$ and unpaired t-test was applied to compare Ctr vs DKO (* $p \leq 0.05, * * p \leq 0.01, * * * p \leq 0.001)$.

B) Relative gene expression of EOMES, TBXT, MESP1, TBX6 obtained from WT hiPSCs, induced or not with CHIR and DMSO or CHIR and Q-VD-OPH for $48 \mathrm{~h}$ and analyzed by qRT-PCR. $\triangle \Delta C t$ values were normalized to un-induced cells. Independent biological repeats are color-coded $(\mathrm{n}=3-4)$. Error bar $=$ Mean +/- S.E.M. Paired t-test was applied to compare $0 \mathrm{~h}$ vs $48 \mathrm{~h}$ and unpaired t-test was applied to compare DMSO vs Q-VD (* $p \leq 0.05, * * p \leq 0.01, * * * p \leq 0.001)$. C) Representative immunofluorescence images of isogenic control (Iso. Ctr) and BAX/BAK DKO hiPSCs stained for T/Bra (top) and EOMES (bottom) and nuclei, before (Oh) and after CHIR treatment. Maximum intensity projections are shown. Scale bar $=50 \mu \mathrm{m}$. Violin plots summarize quantification of the percentage of T/Bra-positive (top) and EOMES-positive (bottom) nuclei after CHIR treatment (Median: plain red line - Quartiles: black dotted lines). Independent biological repeats are color-coded ( $n=3,10-12$ random fields of view/repeat). Mann-Whitney test was applied (**** $p \leq 0.001)$.

D) Immunoblot of isogenic control (Iso. Ctr) and BAX/BAK DKO hiPSCs analyzing T/Bra expression following CHIR addition. Molecular weights (M.W.) are indicated in kDa. E) Immunoblot of WT iPSC treated with CHIR +/- $10 \mu \mathrm{M}$ Q-VD-OPH and analyzed for Nanog expression. Molecular weights (M.W.) are indicated in kDa.

F) Treatment schedule is indicated and timepoints for lysate collection and fixation is depicted by red arrows.

G-H) Immunoblot analysis (G) and representative immunofluorescence images (H) of hiPSCs treated with CHIR +/- $10 \mu \mathrm{M}$ Q-VD-OPH for 24h, then Q-VD-OPH was washed out and cells were incubated for another $28 \mathrm{~h}$ or $48 \mathrm{~h}$ with $\mathrm{CHIR}$ (respectively $52 \mathrm{~h}$ and $72 \mathrm{~h}$ timepoint). 
Figure 5
A
B

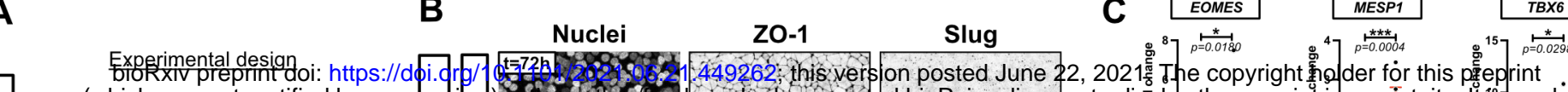
C EOMES
MESP1
TBX6

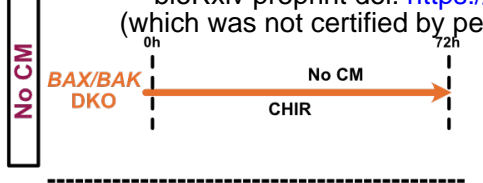

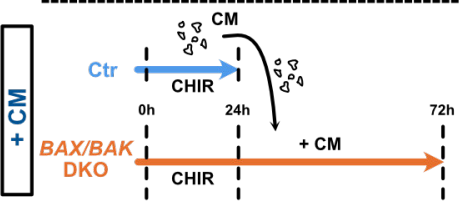
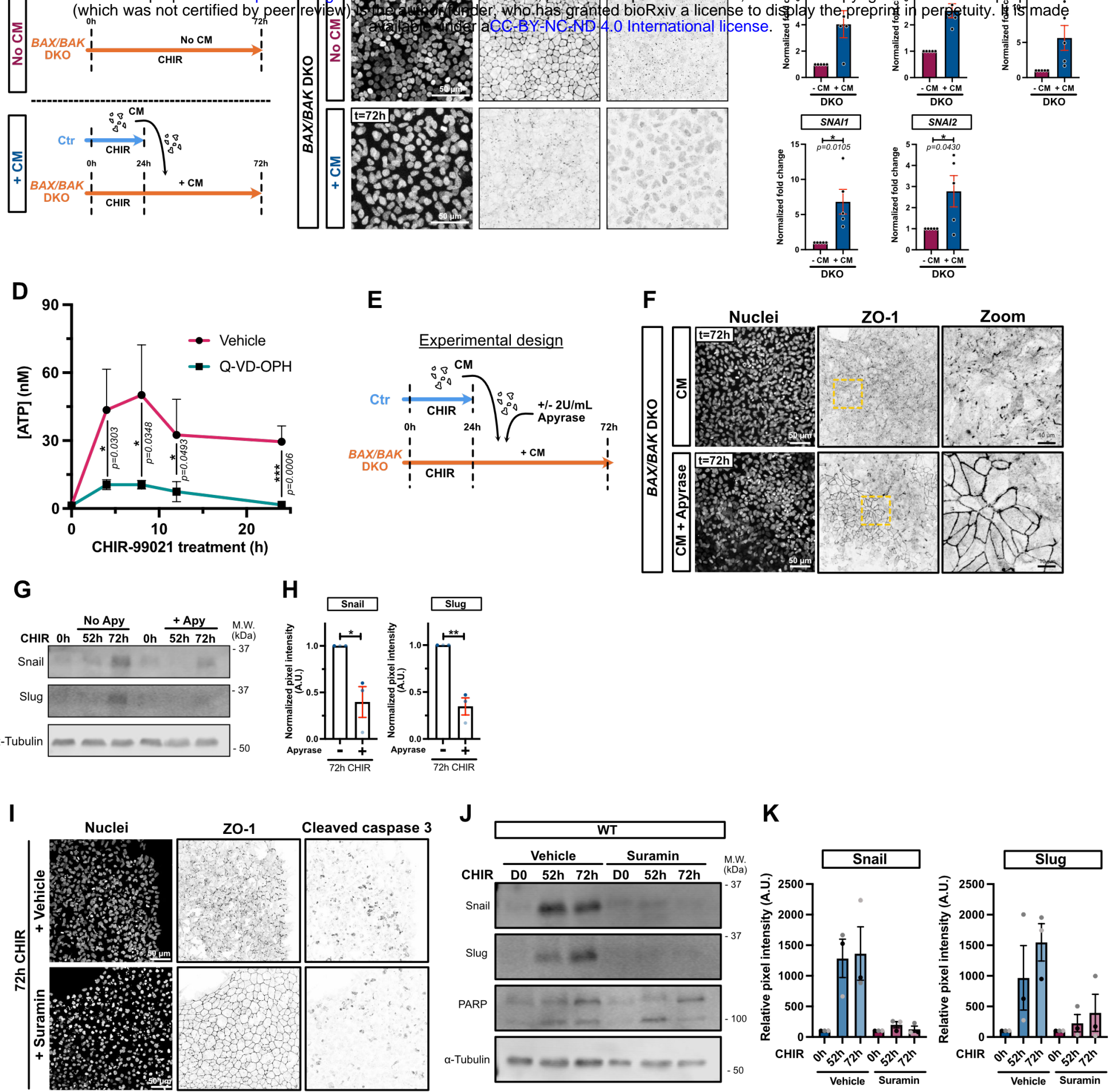

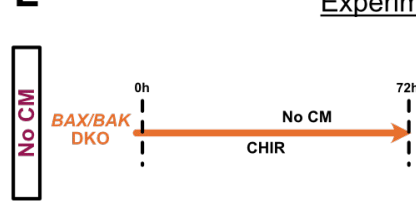

M

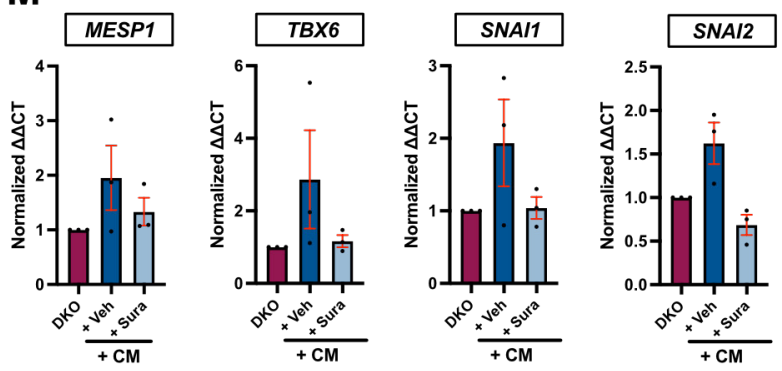

$\mathbf{N}$ Experimental design
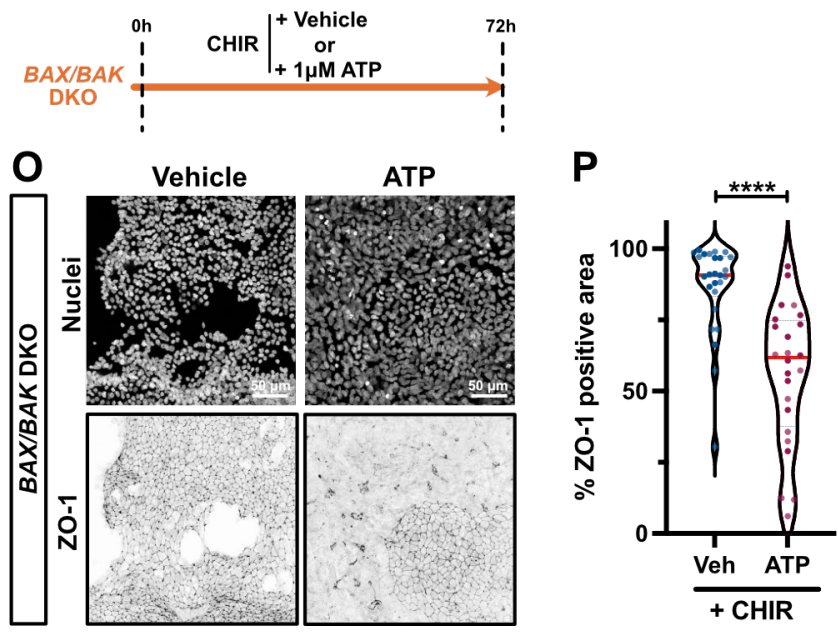


\section{Figure 5}

A) Timeline of conditioned media (CM) experiment.

B) Immunofluorescence pictures of $B A X / B A K$ DKO cells, pre-treated with CHIR for $24 \mathrm{~h}$ and incubated for another $48 \mathrm{~h}$ without (top) or with (bottom) condition media. Cells were fixed at 72h and stained for ZO-1, Slug, and nuclei. Maximum intensity projections are shown. Scale bar $=50 \mu \mathrm{m}$.

C) Relative gene expression of EOMES, MESP1, TBX6, SNAI1 and SNAI2 obtained from BAX/BAK DKO hiPSCs treated as described in A and analyzed by qRT-PCR across 5 independent biological repeats. Fold change in $\Delta \Delta \mathrm{Ct}$ values was normalized to $-\mathrm{CM}$ condition. Error bar $=\mathrm{Mean}+/-$ S.E.M. Paired t test was applied (*p $\leq 0.05, * * * p \leq 0.001)$.

D) Time course of ATP release from dying cells. Luciferase assay was performed on supernatants from hiPSCs treated with CHIR +/- $10 \mu \mathrm{M}$ Q-VD-OPH at indicated timepoints. Luminescence was converted to [ATP] using a standard curve. Three independent biological replicates ( 2 technical replicates each) were plotted as a line graph with mean +/- S.D. Two-way ANOVA with Sidak's multiple comparisons test was applied (* $p \leq 0.05, * * * p \leq 0.001)$.

E) Timeline of apyrase-treated conditioned media (CM) experiment.

F) Representative immunofluorescence pictures of $B A X / B A K$ DKO cells fixed after adding apyrase-treated $\mathrm{CM}$ as depicted in (E). Cells were stained for ZO-1 and nuclei. Maximum intensity projections are shown. Scale bar $=50 \mu \mathrm{m}$. Magnified area (yellow dotted square) is shown for the ZO-1 channel. Scale bar $=10 \mu \mathrm{m}$.

G-H) Immunoblot analysis of WT hiPSCs treated with CHIR +/- 2U/mL Apyrase and probed for Snail, Slug and alpha-Tubulin (G). Normalized expression of Snail and Slug was quantified by densitometry across 3 independent biological replicates (color-coded). Mean +/- S.E.M. Unpaired t-test was applied $(* p \leq 0.05, * * p \leq 0.01)(H)$. I) Immunofluorescence pictures of WT hiPSCs treated with CHIR +/- $100 \mu \mathrm{M}$ Suramin and stained for ZO-1, cleaved-caspase 3 and DNAi. Maximum intensity projections are shown. Scale bar $=50 \mu \mathrm{m}$. 
J-K) Immunoblot analysis of WT hiPSCs treated with CHIR +/- $100 \mu \mathrm{M}$ Suramin and probed for Snail, Slug, PARP and $\alpha$-Tubulin (J). Normalized expression of Snail and Slug was quantified by densitometry across 3 independent biological replicates (color-coded) (K).

L) Timeline of conditioned media (CM) experiment with Suramin.

M) Relative gene expression of MESP1, TBX6, SNAI1 and SNAI2 obtained from BAX/BAK DKO hiPSCs treated as described in K and analyzed by qRT-PCR across 3 independent biological repeats. Mean +/- S.E.M.

N) Timeline of ATP sufficiency experiment O) Immunofluorescence images of $B A X / B A K D K O$ hiPSCs treated with CHIR +/- $1 \mu \mathrm{M}$ ATP and stained for ZO-1 and nuclei. Maximum intensity projections are shown. Scale bar $=50 \mu \mathrm{m}$. P) Violin plot showing percentage of ZO-1 positive areas across 2 independent biological samples ( 3 large images per repeat $(5 \mathrm{~mm} \times 5 \mathrm{~mm}$ ) obtained by tlling). Mann-Whitney test was applied (**** $\mathrm{p} \leq 0.001)$. 


\section{Supp. 1}

bioRxiv preprint doi: https://doi.org/10.1101/2021.06.21.449262; this version posted June 22, 2021. The copyright holder for this preprint A (which was not cantified by peer review) isnthe author/funder, whonhas granted bioRxiv a license to display the preprint in perpetuity. It is made

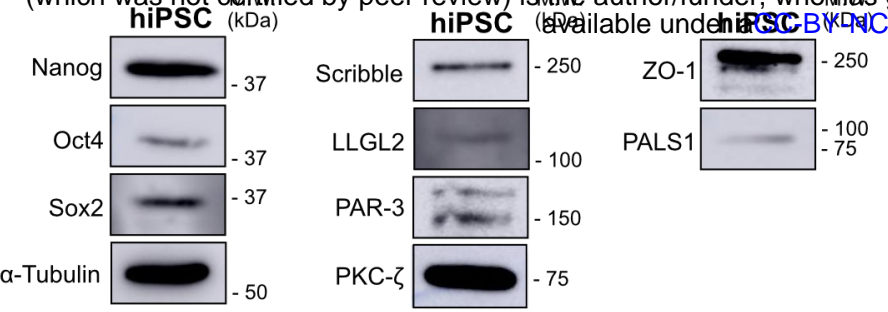

B $\quad$ Repeat 1 Repeat 2 Repeat 3
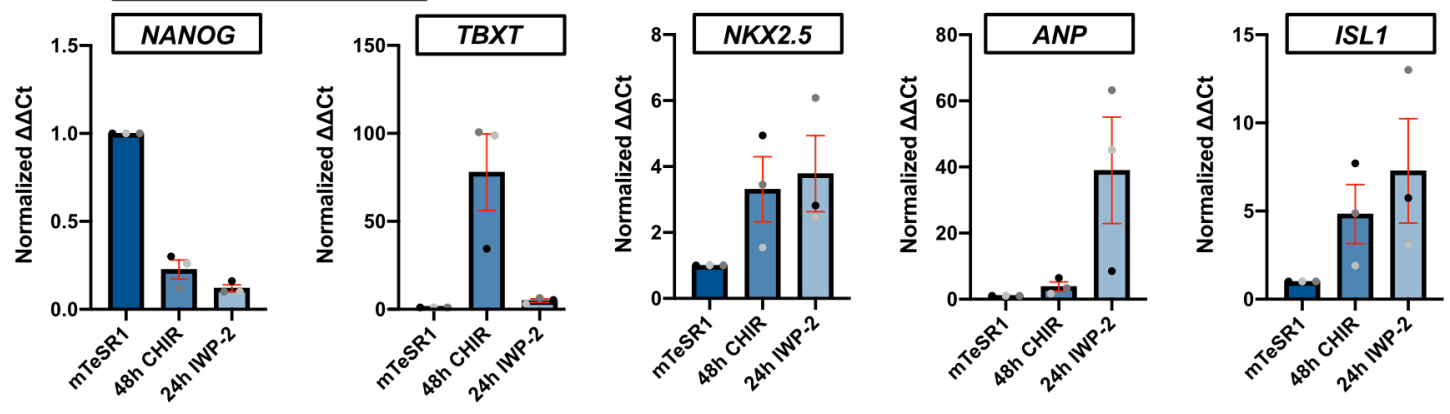

\section{Repeat 1 Repeat 2 Repeat 3}
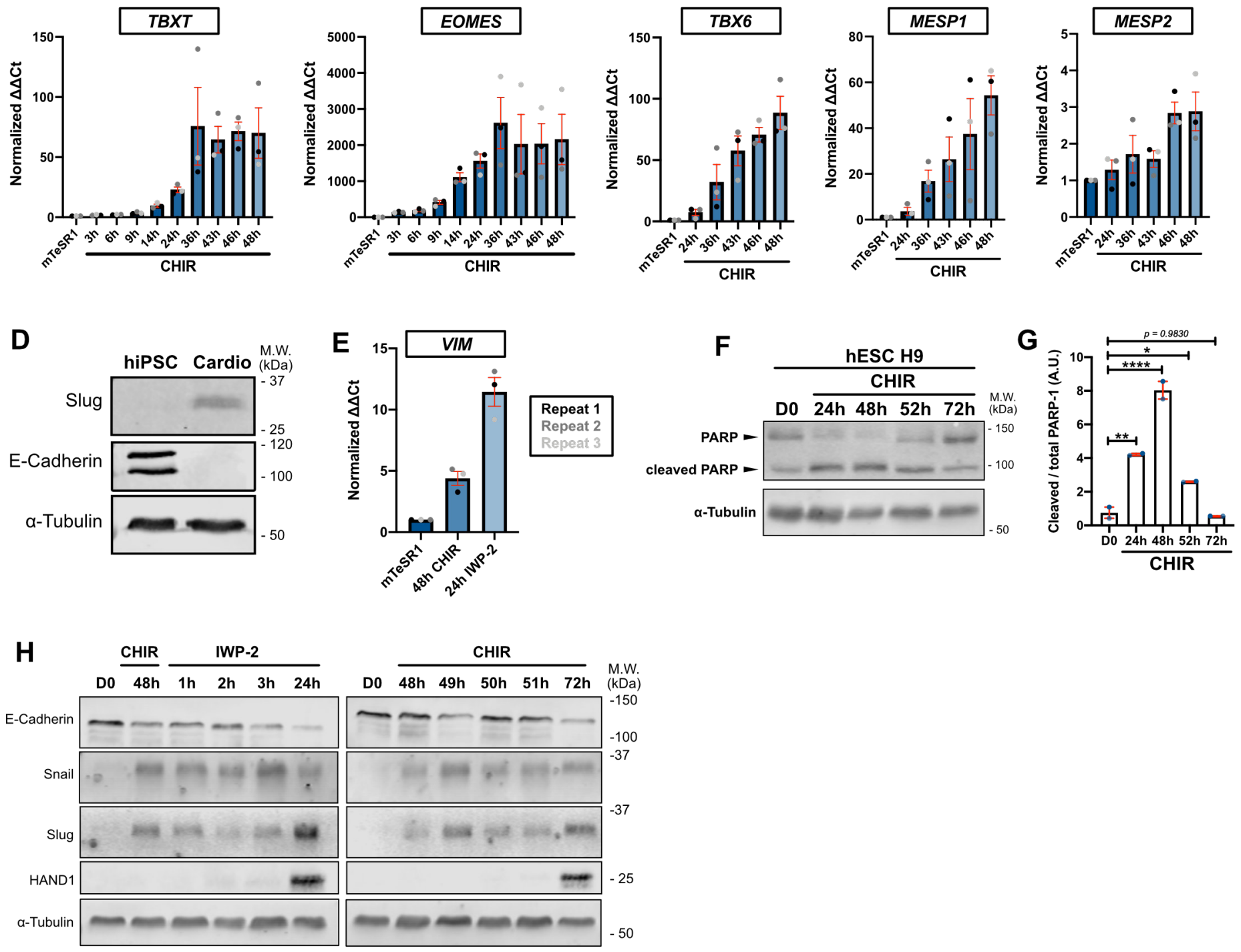


\section{Supp. Fig. 1}

A) Immunoblot of WT hiPSC for pluripotency markers (Nanog, Oct4, Sox2), tight junctions marker (ZO-1), Crumb complex (PALS1), PAR complex (PAR-3, PKC-乙), Scribble complex (LLGL2, Scribble) an $\alpha$-Tubulin. Molecular weights (M.W.) are indicated in kDa.

B) Relative gene expression of NANOG, TBXT, NKX2.5, ANP, ISL1 before (mTeSR1) and during differentiation to mesoderm (CHIR) and cardiac mesoderm (IWP-2). $\Delta \Delta \mathrm{Ct}$ values were normalized to the mTseSR1 condition. Independent biological repeats are color-coded $(n=3)$. Mean +/- S.E.M.

C) Relative gene expression of TBXT, EOMES, TBX6, MESP1, MESP2 before (mTesSR1) and during CHIR induction. $\Delta \Delta \mathrm{Ct}$ values were normalized to the mTseSR1 condition. Independent biological repeats are color-coded $(n=3)$. Mean +/- S.E.M.

D) Immunoblot of hiPSCs and hiPSC-derived cardiomyocytes obtained after applying the differentiation protocol described in Fig. 1B. Expression of EMT markers (Slug and E-Cadherin) were analyzed. Molecular weights (M.W.) are indicated in kDa.

E) Relative gene expression of VIM was analyzed by qRT-PCR during cell conversion. $\Delta \Delta C t$ values were normalized to the mTseSR1 condition. Independent biological repeats are color-coded $(n=3) . M e a n+/-S . E . M$.

F-G) Immunoblot of PARP cleavage in hESC H9 during CHIR treatment. Molecular weights (M.W.) are indicated in kDa (F). PARP cleavage was quantified by densitometry across 2 independent biological repeats (color-coded). Tukey's multiple comparison was applied ( ${ }^{*} p \leq$ $0.05, * * \mathrm{p} \leq 0.01, * * * \mathrm{p} \leq 0.001(\mathrm{G})$.

H) Immunoblot comparing expression of EMT markers (E-Cadherin, Snail, Slug) and cardiac marker (HAND1) between the 2 protocols shown in Fig. $1 \mathrm{~A}$. 


\section{Supp. 3}

A

B
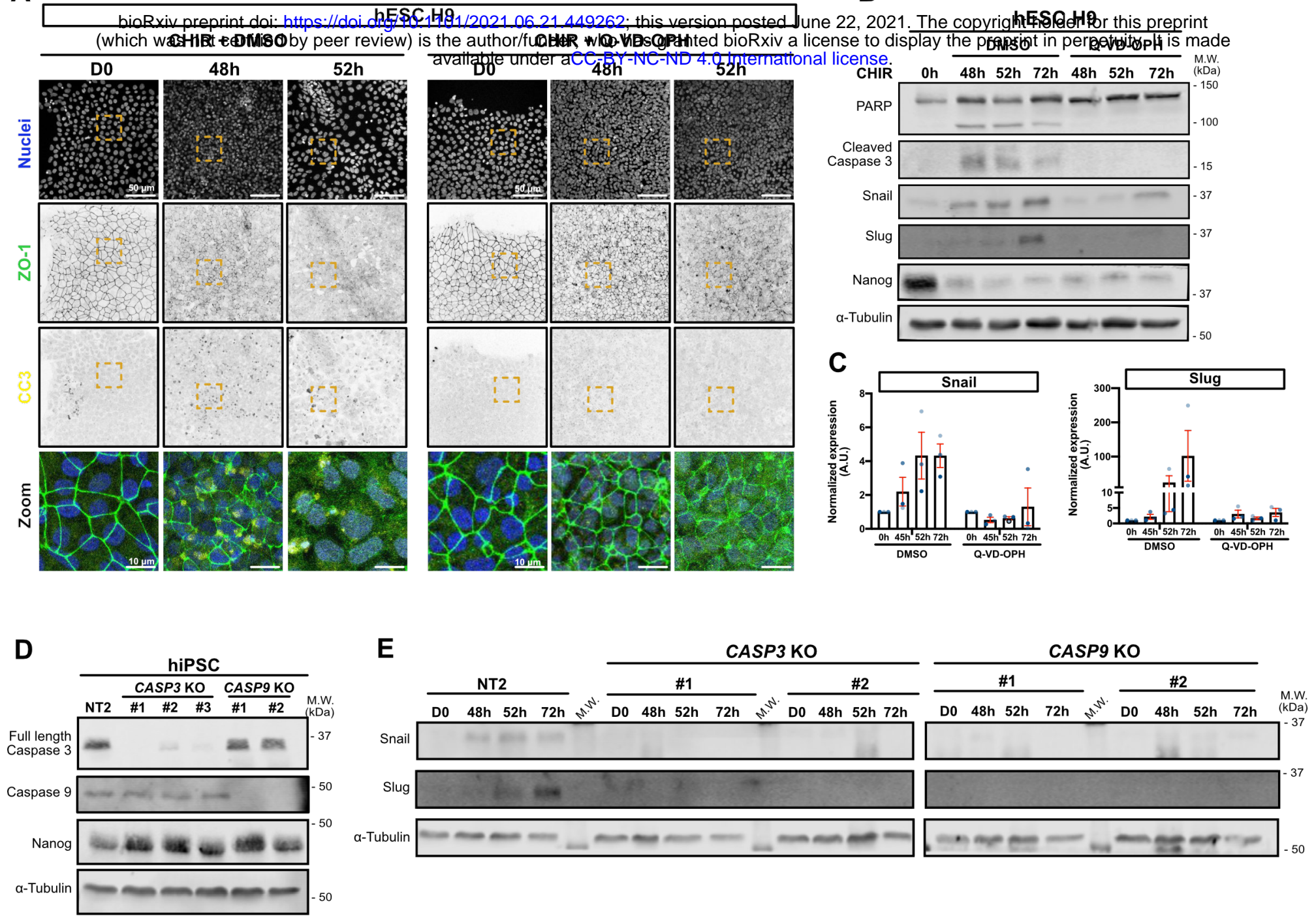

F

72h CHIR

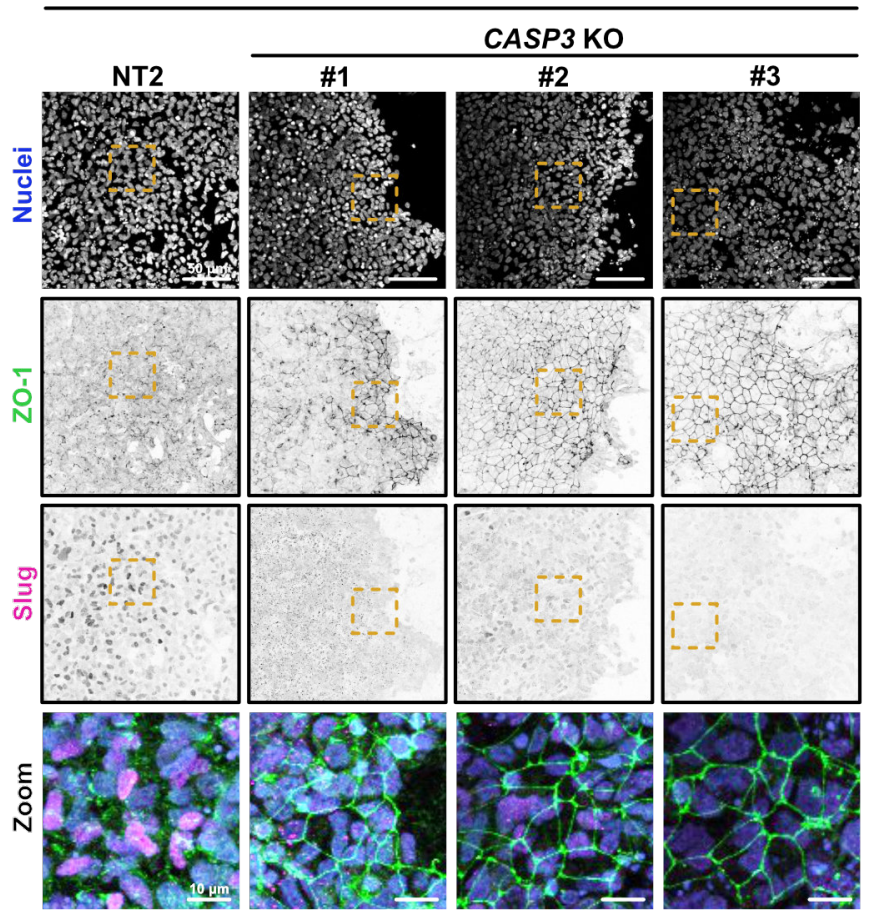

G
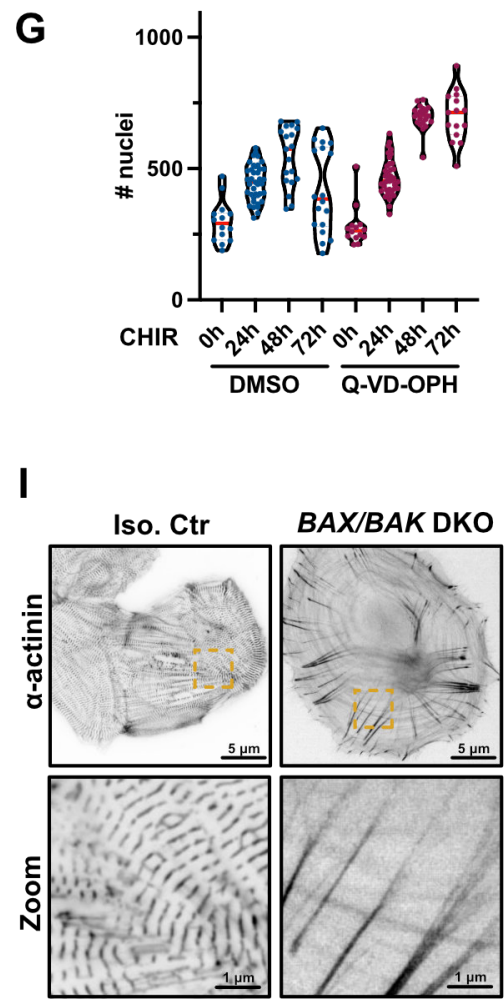

H

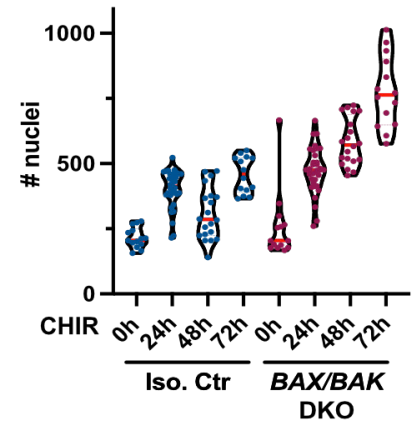

J

CM differentiation protocol
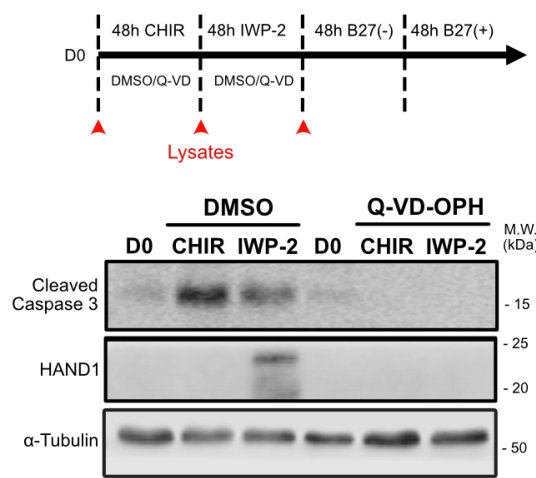
A) Representative immunofluorescence images of hESC H9 co-treated with CHIR and DMSO (left) or CHIR and $10 \mu \mathrm{M}$ Q-VD-OPH (right) stained for ZO-1 (green), cleaved caspase 3 (yellow) and nuclei (blue). Maximum intensity projections are shown. Scale bar $=50 \mu \mathrm{m}$. Magnified area (yellow dotted square) is shown as a merge. Scale bar $=10 \mu \mathrm{m}$.

B-C) Immunoblot analysis of hESC H9 co-treated with CHIR plus DMSO or $10 \mu \mathrm{M}$ Q-VD-OPH. Molecular weights (M.W.) are indicated in kDa (B). Normalized expression of Snail and Slug was quantified by densitometry across 3 independent biological replicates (color-coded). Mean +/S.E.M. (C).

D) Immunoblot of CASP3 and CASP9 KO cell lines (non clonal). Knock-out validation was performed by probing for Caspase 3 and Caspase 9 expression, as well as Nanog as a stem cell marker. Molecular weights (M.W.) are indicated in kDa.

E) Immunoblot of control Non Targeted (NT2) and CASP3 and CASP9 KO cell lines, analyzed for Snail and Slug expression upon CHIR induction. Molecular weights (M.W.) are indicated in kDa. F) Representative immunofluorescence images of Non targeted (NT2) and CASP3 and CASP9 KO hiPSCs, induced 72h with CHIR and stained for ZO-1 (green), Slug (magenta) and nuclei (blue). Maximum intensity projections are shown. Scale bar $=50 \mu \mathrm{m}$. Magnified area (yellow dotted square) is shown as a merge. Scale bar $=10 \mu \mathrm{m}$.

G-H) Violin plots representing numbers of nuclei over time for WT hiPSCs co-treated with CHIR and Q-VD-OPH (G) or BAX/BAK DKO hiPSCs treated with CHIR. (Median: plain red line Quartiles: black dotted lines). Three independent biological repeats (5-12 random fields of view/repeat).

I) Immunofluorescent images of isogenic control and BAX/BAK DKO hiPSC-derived cardiomyocytes, plated at low density and stained for alpha-actinin. Scale bar $=5 \mu \mathrm{m}$. Magnified area (yellow dotted square) is shown. Scale bar $=1 \mu \mathrm{m}$.

J) Immunoblot analysis of hiPSC treated with CHIR and IWP-2 co-treated or not with $10 \mu \mathrm{M}$ QVD-OPH and probed for cleaved caspase 3 and cardiac marker HAND1. Cell lysates were collected as indicated on the timeline (red arrows). 


\section{Supp. 4}

A

DMSO

Q-VD-OPH
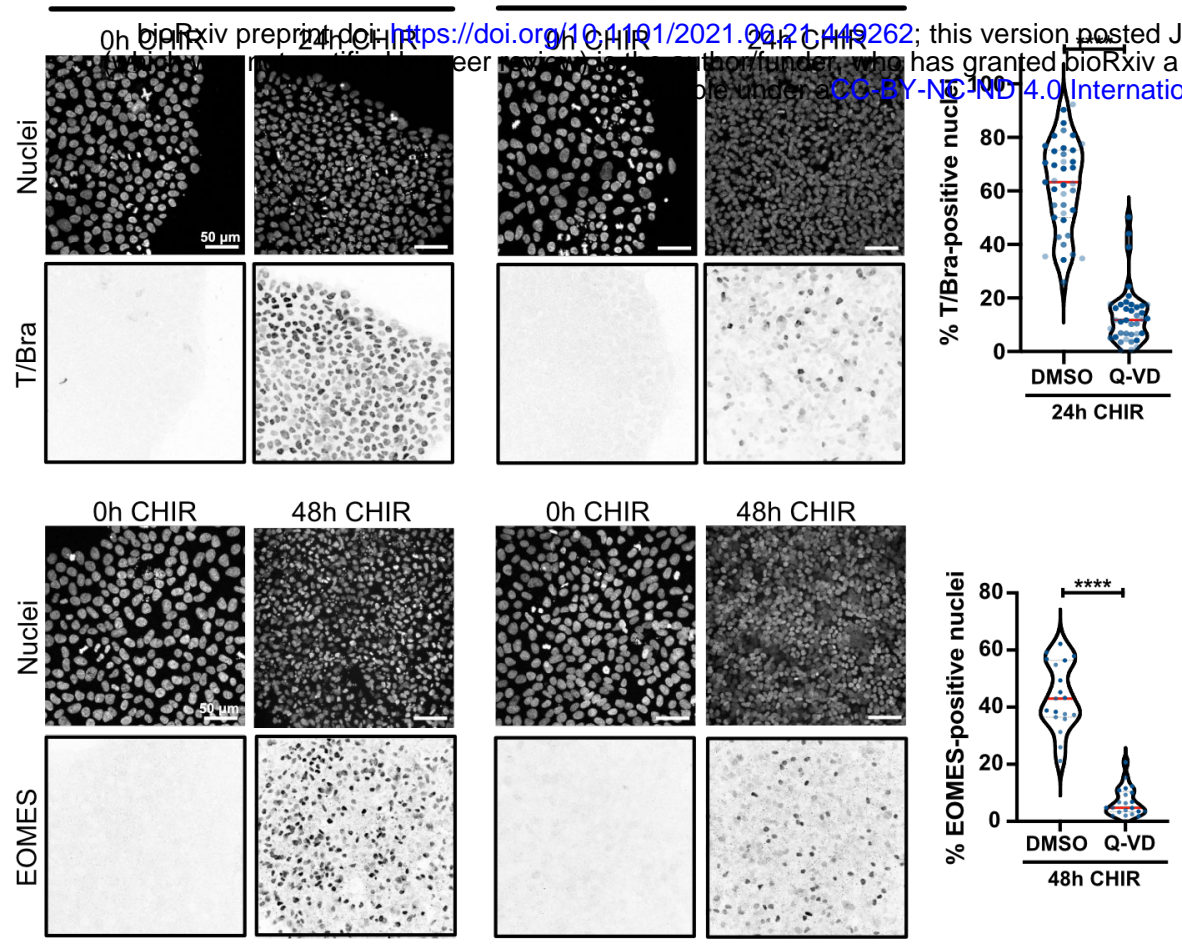

B
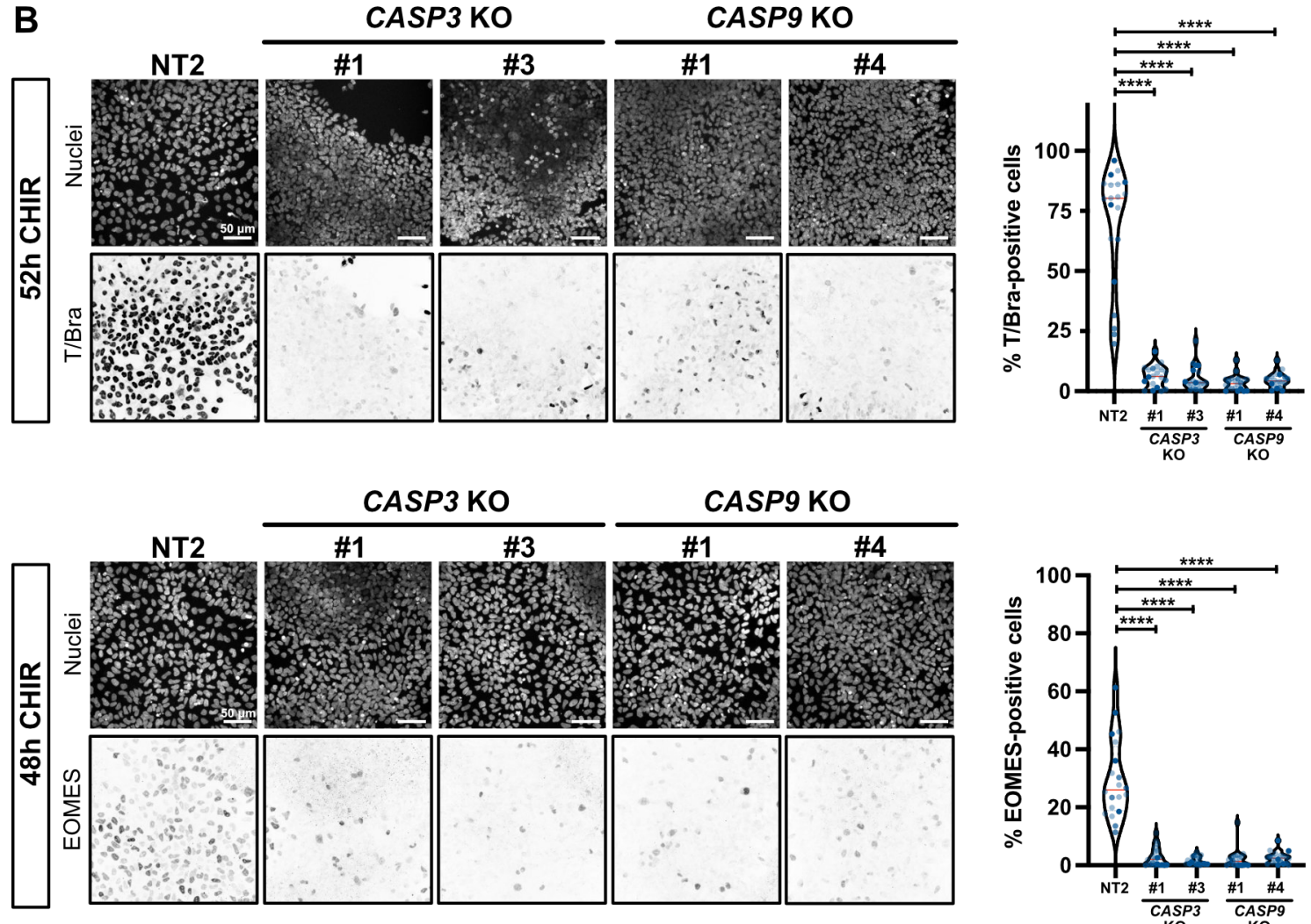

CASP9 KO
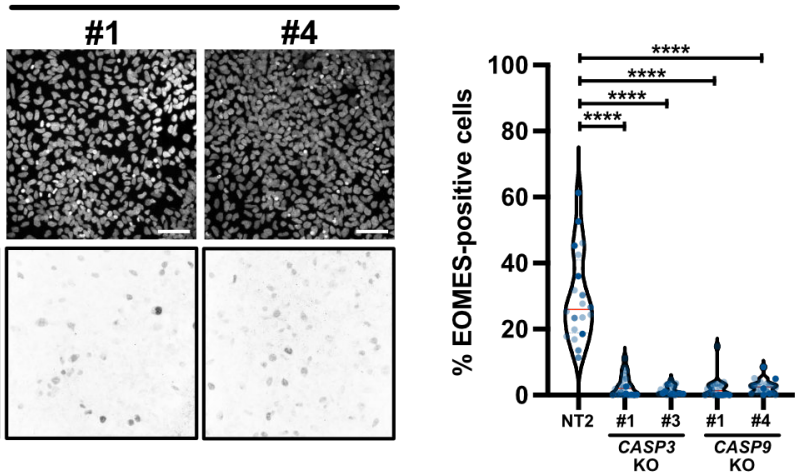

C
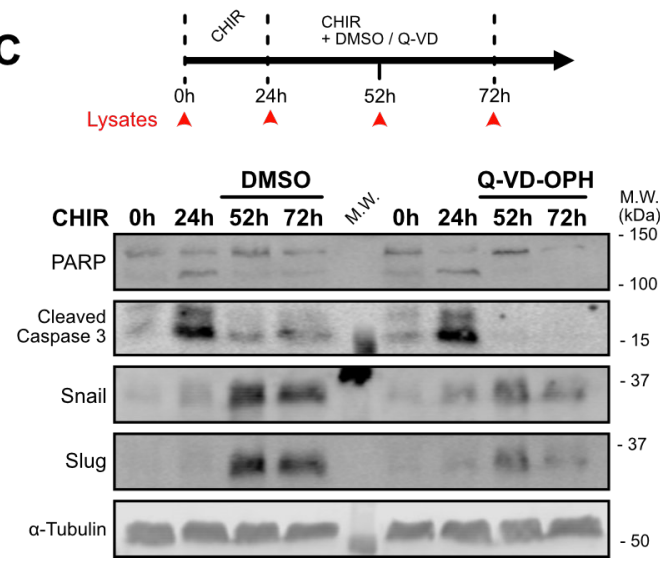

D

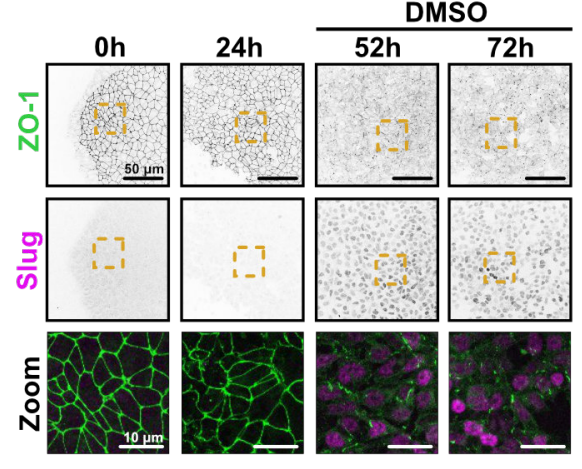

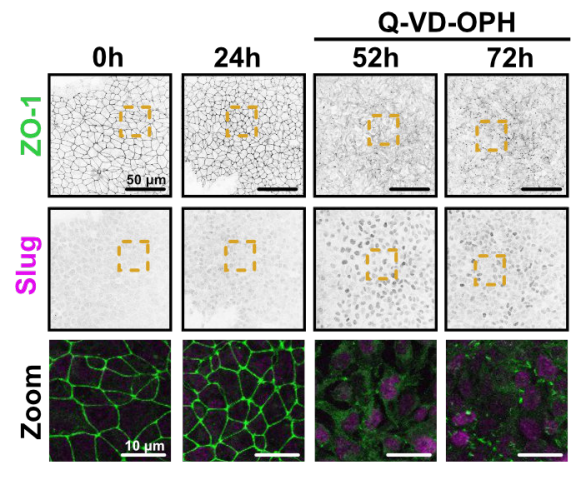




\section{Supp. Fig. 4}

A) Representative immunofluorescence images of WT hiPSCs co-treated with CHIR +/- Q-VD$\mathrm{OPH}$ and stained for T/Bra (top), EOMES (bottom) and nuclei, before (Oh) and after CHIR treatment. Maximum intensity projections are shown. Scale bar $=50 \mu \mathrm{m}$. Violin plots summarize quantification of the percentage of T/Bra-positive (top) and EOMES-positive (bottom) nuclei after CHIR treatment (Median: plain red line - Quartiles: black dotted lines). Independent biological repeats are color-coded $(n=3,10-13$ random fields of view/repeat for T/Bra and $n=2,10$ random fields of view/repeat for EOMES). Mann-Whitney test was applied $(* * * * \mathrm{p} \leq 0.001)$.

B) Representative immunofluorescence images of control NT2, CASP3 and CASP9 KO hiPSCs stained for T/Bra (top), EOMES (bottom) and nuclei after CHIR treatment. Maximum intensity projections are shown. Scale bar $=50 \mu \mathrm{m}$. Violin plots summarize quantification of the percentage of T/Bra-positive (top) and EOMES-positive (bottom) nuclei after CHIR treatment (Median: plain red line - Quartiles: black dotted lines). Independent biological repeats are color-coded ( $n=3,5-7$ random fields of view/repeat. Kurskal-Wallis test was applied (**** $p \leq$ 0.001).

C) Immunoblot analysis of hiPSCs treated with CHIR only for $24 \mathrm{~h}$, before adding CHIR $+/-10 \mu \mathrm{M}$ Q-VD-OPH for another $28 \mathrm{~h}$ and $48 \mathrm{~h}$ with $\mathrm{CHIR}$ (respectively $52 \mathrm{~h}$ and $72 \mathrm{~h}$ timepoint). Treatment timing is indicated on the timeline and lysate collection is depicted by red arrows. ). Molecular weights (M.W.) are indicated in kDa.

D) Representative immunofluorescence images of hiPSCs treated with CHIR for $24 \mathrm{~h}$ before addition of $10 \mu \mathrm{M}$ Q-VD-OPH or DMSO. Cells were cultured for another $28 \mathrm{~h}$ or $72 \mathrm{~h}$ and stained for ZO-1 (green) and Slug (magenta). Maximum intensity projections are shown. Scale bar $=50$ $\mu \mathrm{m}$. Magnified area (yellow dotted square) is shown as a merge (bottom row). Scale bar $=10$ $\mu \mathrm{m}$. 
Supp. 5

A

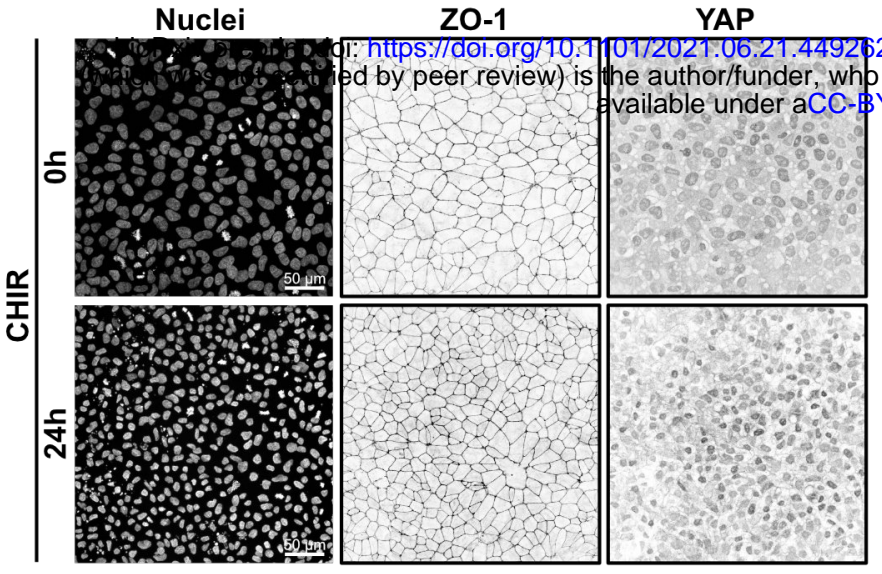

B

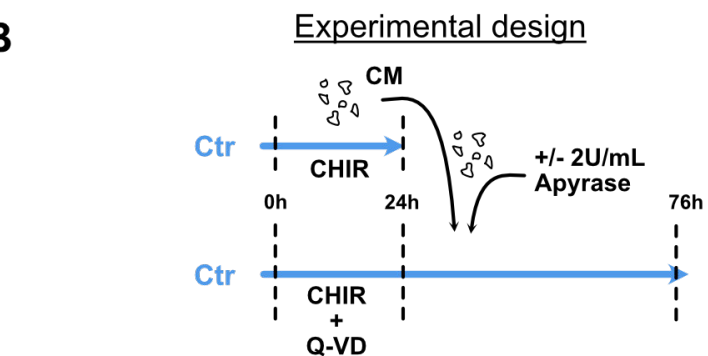

C
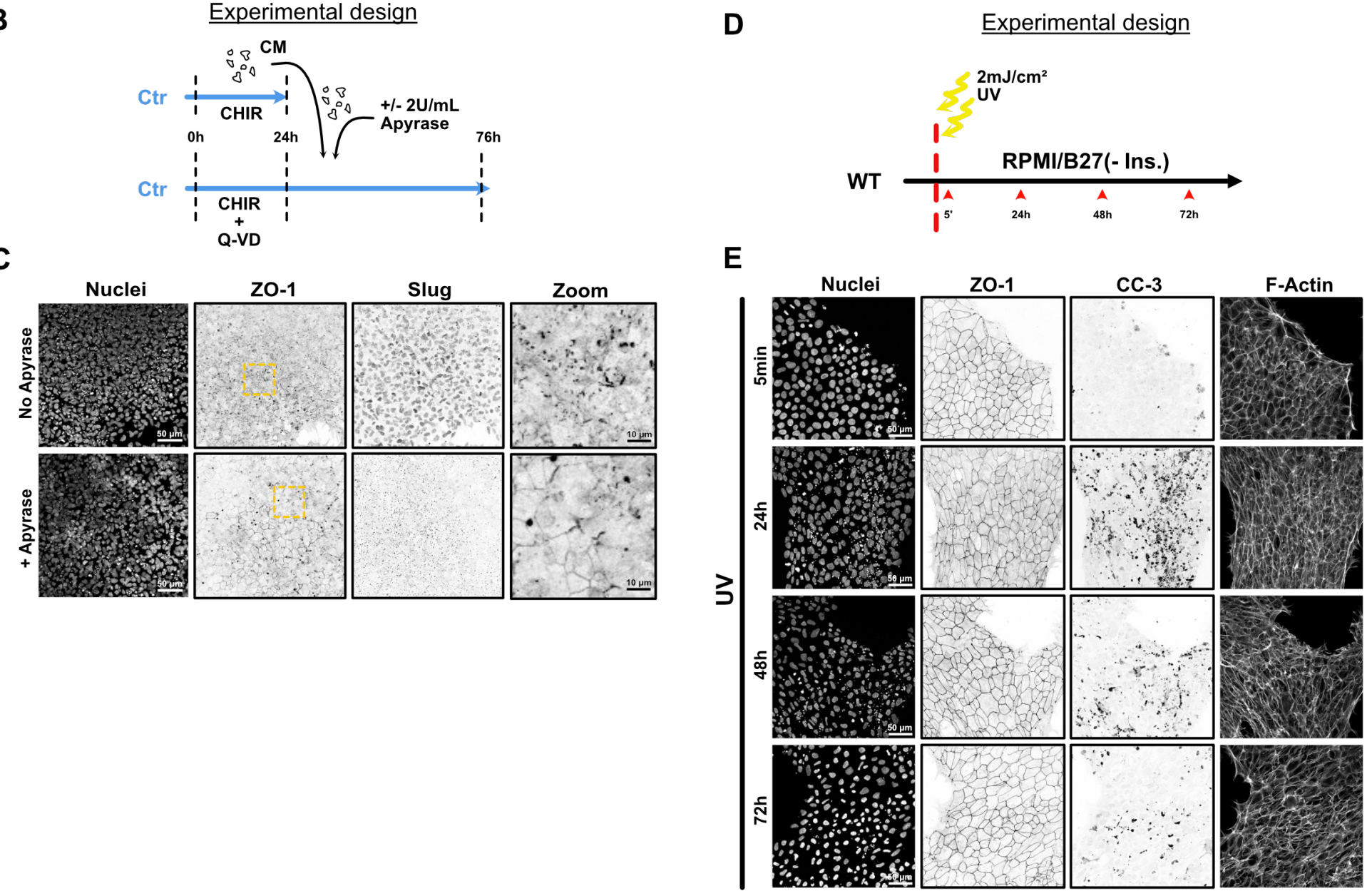

F

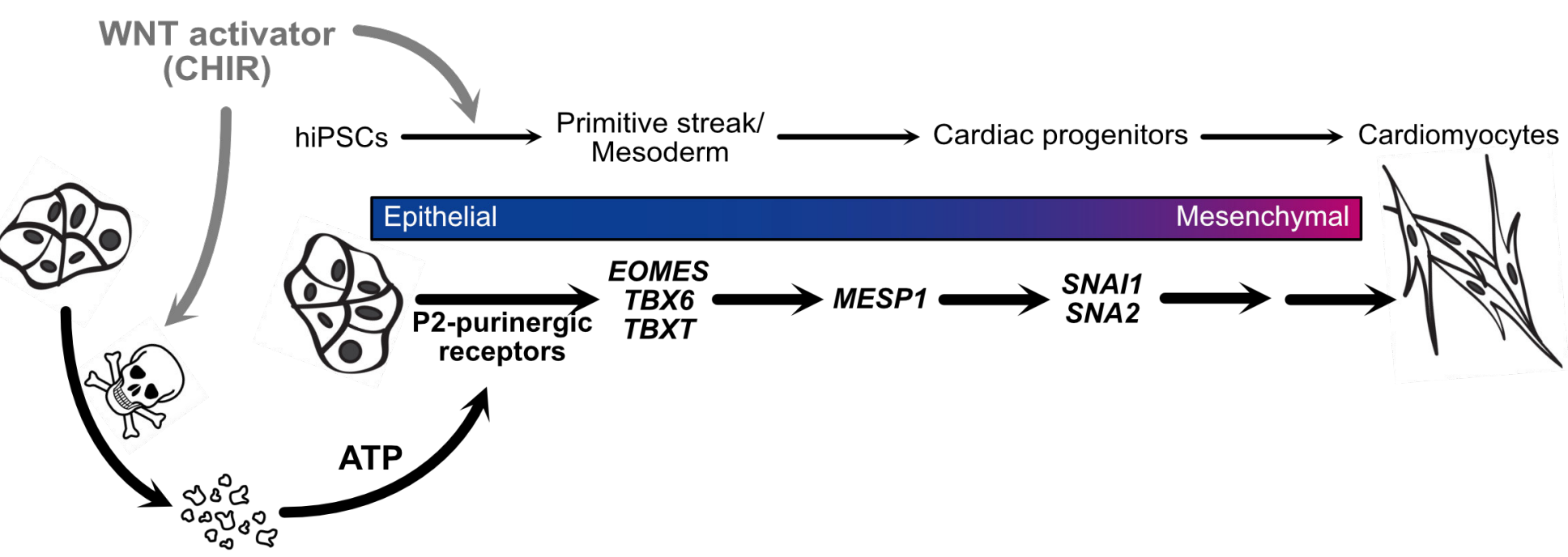




\section{Supp. Fig. 5}

A) Representative immunofluorescence pictures of WT hiPSCs fixed after CHIR treatment and stained for ZO-1, YAP and nuclei. Maximum intensity projections are shown. Scale bar $=50 \mu \mathrm{m}$.

B) Timeline of apyrase-treated conditioned media (CM) experiment.

C) Representative immunofluorescence pictures of WT cells fixed after adding apyrase-treated CM as depicted in (B). Cells were stained for ZO-1, Slug and nuclei. Maximum intensity projections are shown. Scale bar $=50 \mu \mathrm{m}$. Magnified area (yellow dotted square) is shown for the ZO-1 channel. Scale bar $=10 \mu \mathrm{m}$.

D) Timeline for UV exposure. WT hiPSCs were irradiated with $2 \mathrm{~mJ} / \mathrm{cm}^{2}$, kept in RPMI/B27(-Ins) media without CHIR and fixed at the indicated timepoint (red arrowheads).

E) Representative immunofluorescence images from UV-irradiated hiPSC, stained for nuclei, ZO1, cleaved caspase-3 (CC-3) and F-actin. Maximum intensity projections are shown. Scale bar = $50 \mu \mathrm{m}$.

F) Working model 


\section{Table 1: Lab reagents}

\begin{tabular}{|c|c|c|}
\hline Name & Manufacturer & Cat. Number \\
\hline Matrigel & Corning & \# 354277 \\
\hline DMEM/F12 & Gibco & \# 11039-021 \\
\hline mTeSR1 & StemCell Technologies & $\# 85850$ \\
\hline Gentle Cell dissociation & StemCell Technologies & \# 07174 \\
\hline DMEM & Gibco & \# 11965-092 \\
\hline Fetal bovin Serum & Atlantic & \# S11150 \\
\hline Fetal bovin Serum & Peak & \# PS-FB1 \\
\hline Y-27632 & Cayman Chemical & \# 10005583 \\
\hline RPMI 1640 & Gibco & \# 22400089 \\
\hline 50X B27 Minus Insulin (-Ins) & Gibco & \# A1895601 \\
\hline 50X B27 Plus Insulin (+Ins) & Gibco & \# 17504044 \\
\hline IWP-2 & Tocris & $\# 3533$ \\
\hline CHIR-99021 & Tocris & \# 4423 \\
\hline Precision Red & Cytoskeleton & \# ADV02-A \\
\hline Fluoromount-G ${ }^{\mathrm{TM}}$ Slide Mounting Medium & Electron Microscopy Sciences & \# 17984-25 \\
\hline Amicon centrifugal filter unit & EMD Millipore & \# UFC910024 \\
\hline Puromycin & Gibco & \# A11138-03 \\
\hline $35 \mathrm{~mm}$ coverslip dish & MatTek & \# P35G-1.5-10-C \\
\hline $32 \%$ Paraformaldehyde & Electron Microscopy Sciences & $\#$ 157-14-S \\
\hline Rneasy Mini kit & Qiagen & \# 74104 \\
\hline SuperScript III First-Strand Synthesis kit & Invitrogen & \# 12574-018 \\
\hline Maxima SYBR Green/Fluorescein Master Mix & Thermo Fisher Scientic & \# K0242 \\
\hline Annexin V apoptosis kit APC & eBioscience & \# 88-8007 \\
\hline RealTime-Glo Extracellular ATP Assay & Promega & \# GA5010 \\
\hline Apyrase & NED & \# M0398L \\
\hline Suramin hexasodium salt & Tocris & $\# 1472$ \\
\hline ATP & Sigma & \# A1852 \\
\hline
\end{tabular}


bioRxiv preprint doi: https://doi.org/10.1101/2021.06.21.449262; this version posted June 22, 2021. The copyright holder for this preprint (which was not certified by peer review) is the author/funder, who has granted bioRxiv a license to display the preprint in perpetuity. It is made available under aCC-BY-NC-ND 4.0 International license.

Table 2: Antibodies

\begin{tabular}{|c|c|c|c|c|}
\hline Name & Manufacturer & Cat. Number & Application & Dilution \\
\hline \multirow{2}{*}{ E_Cadherin } & Cell Signaling Technologies & \# 3195 & WB & $1: 1000$ \\
\hline & Invitrogen & \# 13-1900 & IF & $1: 500$ \\
\hline \multirow{2}{*}{ Slug } & \multirow{2}{*}{ Cell Signaling Technologies } & \multirow{2}{*}{ \# 9585} & WB & 1:1000 \\
\hline & & & IF & $1: 500$ \\
\hline Snail & Cell Signaling Technologies & \# 3895 & WB & $1: 1000$ \\
\hline Vimentin & Cell Signaling Technologies & \# 5741 & IF & $1: 500$ \\
\hline Zeb1 & Cell Signaling Technologies & \# 3396 & WB & 1:1000 \\
\hline GATA4 & Cell Signaling Technologies & \# 36966 & WB & $1: 1000$ \\
\hline Nanog & Cell Signaling Technologies & \# 4903 & WB & $1: 1000$ \\
\hline Oct4 & Cell Signaling Technologies & \# 75463 & WB & $1: 1000$ \\
\hline Sox2 & Cell Signaling Technologies & \# 3579 & WB & $1: 1000$ \\
\hline Alpha-Tubulin & Sigma-Aldrich & \# T9026 & WB & $1: 3000$ \\
\hline \multirow{2}{*}{ ZO-1 } & \multirow{2}{*}{ Invitrogen } & \multirow{2}{*}{ \# 339100} & WB & $1: 200$ \\
\hline & & & WB & $1: 500$ \\
\hline cTnT & Gift from Prof. Dylan Burnette & & WB & $1: 500$ \\
\hline Nkx2.5 & Cell Signaling Technologies & \# 8792 & WB & $1: 1000$ \\
\hline \multirow{2}{*}{ Cleaved caspase 3} & \multirow{2}{*}{ Cell Signaling Technologies } & \multirow{2}{*}{ \# 9664} & WB & $1: 1000$ \\
\hline & & & IF & $1: 200$ \\
\hline PARP & Cell Signaling Technologies & \# 9542 & WB & $1: 1000$ \\
\hline \multirow{2}{*}{ Scribble } & \multirow{2}{*}{ Santa Cruz Biotechnology } & \multirow{2}{*}{ \# sc-11048 } & WB & $1: 500$ \\
\hline & & & IF & $1: 200$ \\
\hline LLGL2 & Santa Cruz Biotechnology & \# sc-130158 & WB & $1: 500$ \\
\hline \multirow{2}{*}{ PAR-3 } & \multirow{2}{*}{\multicolumn{2}{|c|}{ Homemade }} & WB & $1: 500$ \\
\hline & & & IF & $1: 200$ \\
\hline \multirow{2}{*}{ PKC- $\zeta$} & \multirow{2}{*}{ Santa Cruz Biotechnology } & \multirow{2}{*}{ \# sc-17781 } & WB & $1: 500$ \\
\hline & & & IF & $1: 200$ \\
\hline \multirow{2}{*}{ PALS-1 } & \multirow{2}{*}{\multicolumn{2}{|c|}{ Homemade }} & WB & $1: 500$ \\
\hline & & & IF & $1: 200$ \\
\hline HAND-1 & R \& D Systems & \# AF3168 & WB & $1: 500$ \\
\hline EOMES & Cell Signaling Technologies & \# 81493 & IF & $1: 200$ \\
\hline \multirow[b]{2}{*}{ T/Bra } & \multirow{2}{*}{ abcam } & \multirow{2}{*}{ \# ab209665 } & IF & $1: 200$ \\
\hline & & & WB & $1: 1000$ \\
\hline alpha actinin 2 & Signa & A7811 & IF & $1: 500$ \\
\hline YAP & Novus & \# NB110-58358 & IF & $1: 200$ \\
\hline
\end{tabular}

Table 2: Secondary Antibodies

\begin{tabular}{|c|c|c|c|c|}
\hline $\begin{array}{l}\text { Alexa Fluor } 680 \text { donkey anti- } \\
\text { mouse IgG }(H+L)\end{array}$ & Invitrogen & \# A10038 & WB & 1:10000 \\
\hline $\begin{array}{l}\text { Goat anti-Rabbit (H\&L), } \\
\text { DyLight } 8004 \mathrm{X} \text { PEG } \\
\text { conjugate }\end{array}$ & Invitrogen & \# SA535571 & WB & 1:10000 \\
\hline $\begin{array}{l}\text { Alexa Fluor } 594 \text { goat anti- } \\
\text { rabbit IgG }(\mathrm{H}+\mathrm{L})\end{array}$ & Invitrogen & \# A11037 & IF & 1:500 \\
\hline $\begin{array}{l}\text { Alexa Fluor } 488 \text { goat anti- } \\
\text { mouse } \lg G(\mathrm{H}+\mathrm{L})\end{array}$ & Invitrogen & \# A11029 & IF & 1:500 \\
\hline $\begin{array}{l}\text { Alexa Fluor } 488 \text { goat anti- } \\
\text { rat } \lg G(\mathrm{H}+\mathrm{L})\end{array}$ & Invitrogen & \# A11006 & IF & $1: 500$ \\
\hline
\end{tabular}

\begin{tabular}{|l|l|l|l|l|}
\hline \multicolumn{3}{|c|}{ Table 2: Others } \\
\hline Alexa Fluor 647 Phalloidin & Invitrogen & \# A22287 & IF & $1: 500$ \\
\hline Hoechst 33342 & Invitrogen & \# H1399 & IF & $1: 500$ \\
\hline
\end{tabular}




\section{Table 3: CRISPR-Cas9 sgRNA *}

\begin{tabular}{|c|c|c|c|}
\hline Name & Sequence (5'->3') & \multicolumn{2}{|c|}{ Comment } \\
\hline H.s. MESP1 \#1 Fwd & CACCGCGAGTCCTGGATGCTCTCTG & & \\
\hline H.s. MESP1 \#1 Rev & aaacCAGAGAGCATCCAGGACTCGc & & \\
\hline H.s. MESP1 \#2 Fwd & CACCGAGACACGGACGCAGGCTGAG & & \\
\hline H.s. MESP1 \#2 Rev & aaacCTCAGCCTGCGTCCGTGTCTc & & \\
\hline H.s. MESP1 \#3 Fwd & CACCGGCCGTGCTAGGCCTCAGCG & & \\
\hline H.s. MESP1 \#3 Rev & aаacCGCTGAGGCCTAGCACGGCc & & \\
\hline H.s. MESP2 \#1 Fwd & CACCGACGGGGGCGACTGTATCTTG & & \\
\hline H.S. MESP2 \#1 Rev & aaacCAAGATACAGTCGCCCCCGTc & & \\
\hline H.s. MESP2 \#2 Fwd & CACCGTCCCTTGGGACGAATACGG & & \\
\hline H.s. MESP2 \#2 Rev & aaacCCGTATTCGTCCCAAGGGAc & & \\
\hline H.s. CASP3 \#1 Fwd & CACCGAGTTTCTGAATGTTTCCCTG & & \\
\hline H.s. CASP3 \#1 Rev & aaacCAGGGAAACATTCAGAAACTc & & \\
\hline H.s. CASP3 \#2 Fwd & CACCGTGTCGATGCAGCAAACCTCA & & \\
\hline H.s. CASP3 \#2 Rev & aаacTGAGGTTTGCTGCATCGACAc & & \\
\hline H.s. CASP3 \#3 Fwd & CACCGGAAGCGAATCAATGGACTC & & \\
\hline H.S. CASP3 \#3 Rev & aaacGAGTCCATTGATTCGCTTCC & & \\
\hline H.s. CASP9 \#1 Fwd & CACCGTTCAGGCCCCATATGATCG & & \\
\hline H.s. CASP9 \#1 Rev & aaacCGATCATATGGGGCCTGAAc & & \\
\hline H.s. CASP9 \#4 Fwd & CACCGCTACTCGCCATGGACGAAG & & \\
\hline H.s. CASP9 \#4 Rev & aаacCTTCGTCCATGGCGAGTAGc & & \\
\hline Hs SNAI1 Ex1.3 Fwd & CACCGTGTAGTTAGGCTTCCGATTG & DKO \#1 & DKO \#3 \\
\hline Hs SNAI1 Ex1.3 Rev & aаacCAATCGGAAGCCTAACTACAc & & \\
\hline Hs SNAI1 Ex2.1 Fwd & CACCGGATGAGCATTGGCAGCGAGG & 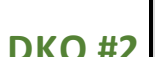 & \\
\hline Hs SNAI1 Ex2.1 Rev & aаacCCTCGCTGCCAATGCTCATCc & & \\
\hline Hs SNAI2 Ex2.1 Fwd & CACCGGCTGTAGTTTGGCTTTTTGG & DKO \#1 & \\
\hline Hs SNAI2 Ex2.1 Rev & aаacCCAAAAAGCCAAACTACAGCc & & \\
\hline Hs SNAI2 Ex2.2 Fwd & CACCGGAAATGCTTCTTGACCAGGA & \#21 & \\
\hline Hs SNAI2 Ex2.2 Rev & aаacTCCTGGTCAAGAAGCATTTCc & & \\
\hline Non Target (NT2) Fwd & CACCGACGTGTAAGGCGAACGCCTT & & \\
\hline Non Target (NT2) Rev & aaacAAGGCGTTCGCCTTACACGTc & & \\
\hline
\end{tabular}

* BsmBI overhang

* Target sequence 


\section{Table 4: RT-qPCR primers}

\begin{tabular}{|c|c|c|}
\hline Name & Sequence (5'->3') & References \\
\hline H.s. MESP1 Fwd & CGTCAGTTGTCCCTTGTCACTT & \\
\hline H.s. MESP1 Rev & GCTGGCTCTGTTGGAGACCT & \\
\hline H.s. MESP2 Fwd & GGCTTCCCTCTTTCCATCCA & \\
\hline H.s. MESP2 Rev & GGAGCCTTGGCTAAAGGAGA & \\
\hline H.s. Bra/T Fwd & TTTCCAGATGGTGAGAGCCG & \multirow{6}{*}{ http://dx.doi.org/10.1101/665695 } \\
\hline H.s. Bra/T Rev & CCGATGCCTCAACTCTCCAG & \\
\hline H.s. Nanog Fwd & CCCAAAGGCAAACAACCCACTT & \\
\hline H.s. Nanog Rev & AGCTGGGTGGAAGAGAACACA & \\
\hline H.s. Vimentin Fwd & AGTCCACTGAGTACCGGAGAC & \\
\hline H.s. Vimentin Rev & CATTTCACGCATCTGGCGTTC & \\
\hline H.s. Isl1 Fwd & TTTATTGTCGGAAGACTTGCCACTT & \multirow{6}{*}{2018 10.7554/eLife.31706 } \\
\hline H.s. IsI1 Rev & TCAAAGACCACCGTACAACCTTTATCT & \\
\hline H.s. Nkx2.5 Fwd & ACCGATCCCACCTCAACAGC & \\
\hline H.s. Nkx2.5 Rev & CTCCGCAGGAGTGAATGCAA & \\
\hline H.s. ANP/NPPA Fwd & GCTGCAGCTTCCTGTCAACACT & \\
\hline H.s. ANP/NPPA Rev & AGGCGAGGAAGTCACCATCAA & \\
\hline H.s. EOMES Fwd & ATCATTACGAAACAGGGCAGGC & \\
\hline H.S. EOMES Rev & CGGGGTTGGTATTTGTGTAAGG & \\
\hline H.s. SNAI1 Fwd & GCTGCAGGACTCTAATCCAGAGTT & \\
\hline H.s. SNAI1 Rev & GACAGAGTCCCAGATGAGCATTG & \\
\hline H.s. SNAI2 Fwd & AGATGCATATTCGGACCCAC & \\
\hline H.s. SNAI2 Rev & CCTCATGTTTGTGCAGGAGA & \\
\hline H.s.. Sox1 Fwd & ATGCACCGCTACGACATGG & \multirow{2}{*}{ doi: $10.1038 /$ nbt1163 } \\
\hline H.S.. Sox1 Rev & CTCATGTAGCCCTGCGAGTTG & \\
\hline H.S.. Sox17 Fwd & GGCGCAGCAGAATCCAGA & \multirow{6}{*}{ https://doi.org/10.1016/j.stem.2009.11.015 } \\
\hline H.S.. Sox17 Rev & CCACGACTTGCCCAGCAT & \\
\hline H.s.. Sox2 Fwd & TGGACAGTTACGCGCACAT & \\
\hline H.S.. Sox2 Rev & CGAGTAGGACATGCTGTAGGT & \\
\hline H.s. SIP1 Fwd & CGCTTGACATCACTGAAGGA & \\
\hline H.s. SIP1 Rev & CTTGCCACACTCTGTGCATT & \\
\hline H.s. GAPDH Fwd & GGACCTGACCTGCCGTCTAGAA & \\
\hline H.s. GAPDH Rev & GGTGTCGCTGTTGAAGTCAGAG & \\
\hline
\end{tabular}




\section{Movie legends}

\section{Supplementary movie 1}

Phase contrast timelapse of hiPSC-derived cardiomyocytes obtained using the GiWi differentiation protocol. Spontaneous beating was observed 12 days after protocol initiation and immature cardiomyocytes were maintained in RPMI/B27 (+ Ins.).

\section{Supplementary movie 2}

Timelapse imaging of mEGFP-TJP1 knock-in hiPSCs, starting 40h after CHIR-99021 treatment. Scale bar $=50 \mu \mathrm{m}$. Maximum intensity projections are shown.

\section{Supplementary movie 3}

Phase contrast timelapse of control (NT2) and MESP1 knockout (\#1 and \#2) hiPSC-derived cardiomyocytes. Movies were recorded 12 days after GiWi protocol initiation using an EVOS FL microscope (Obj. x10). Scale bar $=400 \mu \mathrm{m}$.

\section{Supplementary movie 4}

Phase contrast timelapse of control (Ctr) and BAX/BAK DKO hiPSC-derived cardiomyocytes. Movies were recorded 12 days after GiWi protocol initiation using an EVOS FL microscope (Obj. x10). Scale bar $=400 \mu \mathrm{m}$. 